\title{
From 2D hyperbolic forests to 3D Euclidean entangled thickets
}

\author{
S.T. Hyde ${ }^{1}$ and C. Oguey ${ }^{2, \text { a }}$ \\ 1 Applied Mathematics, Research School of Physical Sciences, Australian National University, Canberra, A.C.T. 0200, Australia \\ ${ }^{2}$ LPTM $^{\mathrm{b}}$, Université de Cergy Pontoise, 5 Mail G. Lussac, 95031 Cergy-Pontoise, France
}

Received 10 December 1999

\begin{abstract}
A method is developed to construct and analyse a wide class of graphs embedded in Euclidean 3D space, including multiply-connected and entangled examples. The graphs are derived via embeddings of infinite families of trees (forests) in the hyperbolic plane, and subsequent folding into triply periodic minimal surfaces, including the $P, D$, gyroid and $H$ surfaces. Some of these graphs are natural generalisations of bicontinuous topologies to bi-, tri-, quadra- and octa-continuous forms. Interwoven layer graphs and periodic sets of finite clusters also emerge from the algorithm. Many of the graphs are chiral. The generated graphs are compared with some organo-metallic molecular crystals with multiple frameworks and molecular mesophases found in copolymer melts.
\end{abstract}

PACS. 61.50.Ah Theory of crystal structure, crystal symmetry; calculations and modeling - 61.25.Hq Macromolecular and polymer solutions; polymer melts; swelling - 61.30.Cz Theory and models of liquid crystal structure

\section{Introduction}

Infinite crystalline 3D graphs, consisting of translationally ordered arrangements of points (vertices) and lines joining them (edges), are relevant to crystalline condensed materials, including "hard" atomic and molecular crystals and soft liquid crystals. Yet systematic derivation of these graphs remains surprisingly undeveloped, with some notable exceptions [1-7]. There are many possible graphs in 3D Euclidean space, $E^{3}$, not all of which are relevant to condensed matter. In order to decide the controlling factors governing atomic or molecular assembly, it is useful to have at hand a fuller catalogue of 3D graphs, including networks of disjoint graphs. Here we discuss one route towards denumeration of translationally symmetric graphs in $E^{3}$, introduced in [8]. The technique delivers a variety of non-trivial topological and geometric solutions, including interwoven infinite graphs that are generalisations of the pairs of interwoven 3D graphs found in molecular crystals [9] and bicontinuous (meso)phases [10-13]. These structures are applicable to novel polycontinuous molecular crystal and liquid crystal phases. 3D arrays of interwoven disjoint 2D (mesh) structures, and arrays of finite graphs (or clusters) are also generated by the process.

Two general features constraining possible graphs will be imposed here. 1) Spatial homogeneity under its most stringent form. All the patterns studied here are periodic in the three independent directions of space. 2) Local regularity, which is a loose way to demand a natural

\footnotetext{
${ }^{a}$ e-mail: oguey@ptm.u-cergy.fr

b CNRS ESA 8089
}

cutoff at short length scales. Depending on the system at hand, this UV limit is due to e.g. stability limits of the (meso)phase under study, bonding, hard-core or other microscopic interactions, effective contributions involving the curvatures, bending or other geometric parameters. Ultimately, of course, these constraints will not guarantee the physical relevance of a graph. But they do afford a sensible starting point for the search for physically relevant graphs.

We accordingly confine our geometric analysis to very symmetric graphs, generated by particular decorations of triply periodic minimal surfaces (TPMS) with 2D hyperbolic networks. We choose four TPMS of genus three (per unit cell): Schoen's gyroid $(G)$, Schwarz' $P$ and $D$ surfaces (all three cubic) and the $H$ surface (hexagonal) [13-15]. A fifth TPMS, the (cubic) $I-W P$ surface of genus four [16], is also mentioned briefly, to demonstrate the broader applicability of the technique. The graphs are mostly "Platonic" or "regular", in that all vertices are identically disposed with respect to arrangements of edges and neighbouring vertices and all vertex angles and edges are equal. We control some aspects of the graphs by design within the hyperbolic plane $\left(H^{2}\right)$; their final geometry and topology is dependent on the embedding of the (slightly distorted) 2D structure in $E^{3}$. Within $H^{2}$, the structure consists of disjoint close-packed trees, that we call a forest. Once a forest is embedded in $E^{3}$, branches of the trees fuse, forming 3D crystalline nets that remain closepacked in a specific sense we make clear in the paper. We call these 3D arrangements thickets. The analysis does allow for generalisation to regular graphs on non-minimal 
surfaces, though minimal surfaces have a number of interesting features: balance (mean curvature $H=$ 0 ), hyperbolicity, homogeneous embedding in space, smoothness and explicit parametrisation through the Weierstrass-Enneper formula [14,15].

The paper is organised as follows. First we recall some basic geometry of $2 \mathrm{D}$ hyperbolic space. We then outline properties of graphs embedded in $H^{2}$. We focus our attention on regular graphs and show how to embed an unlimited number of trees in $H^{2}$. A mapping from $H^{2}$ to $E^{3}$ is introduced, that derives from analysis of the simpler TPMS in $E^{3}$. That mapping is used to generate examples of thickets in $E^{3}$, that are arrays of disjoint three-, fourand six-coordinated graphs. In closing the paper, we discuss the relevance of the examples generated to condensed atomic and molecular systems.

\section{The hyperbolic plane, $\mathrm{H}^{2}$}

There are a number of models of 2D hyperbolic geometry. For convenience we use Poincaré's conformal model of $H^{2}$ in the unit disc $\left(D^{2}\right.$, whose boundary is the unit circle, $S^{1}$ ) [17]. The metric is radially symmetric about the origin of $D^{2}$. The hyperbolic arc length $\mathrm{d} s$ is related to the Euclidean arc length $\mathrm{d} r$ in the disc by

$$
|\mathrm{d} s|=\frac{2|\mathrm{~d} r|}{1-r^{2}}
$$

where $r$ is the radial coordinate. There is severe nonlinearity in the metric of the Poincaré disc model, so that $S^{1}$ represents the points at infinity of $H^{2}$.

\subsection{Geodesics}

Geodesics of $H^{2}$ are represented in the Poincaré disc by circular arcs that intersect $S^{1}$ orthogonally. They can be intersecting or parallel but they can also be ultraparallel. Within the model, the parallel geodesics meet on $S^{1}$, while ultraparallel ones do not intersect anywhere on $D^{2}$ (Fig. 1).

\subsection{Rotations and translations}

The set of hyperbolic motions (transformations preserving the metrics of $H^{2}$ ) forms a continuous and non-commutative group. The operations preserving the orientation of $H^{2}$ include rotations (characterised by a single fixed point, the centre of rotation) and translations (leaving two points of the unit circle fixed in the Poincaré representation). The improper motions (reversing orientation) are mirror reflections composed with any orientation preserving transformation.

The rotations around a fixed centre form a continuous subgroup of $H^{2}$. The trajectory of any point under this group is a circle, or cycle. The set of translations with two given fixed points at infinity also form a one-parameter subgroup. The trajectory of a point $x$ under this group is a geodesic only if $x$ lies in the (unique) geodesic connecting

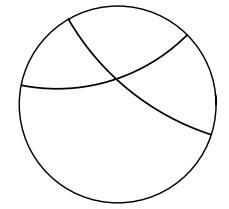

(a)

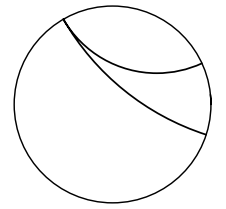

(b)

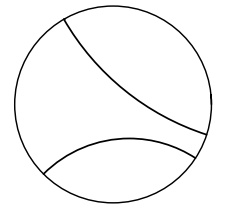

(c)
Fig. 1. (a) Intersecting, (b) parallel and (c) ultraparallel geodesics in $H^{2}$.

the two fixed points. This guiding geodesic, as we call it, is the only one left globally invariant by the translation. Otherwise (that is, generically), the trajectory is an arc of circle (in the Poincaré model) called a hypercycle. The intermediate case, when the transformation leaves a single point fixed at infinity, is a horocycle.

Contrary to $E^{2}$, homogeneous dilatation is not a similarity in $H^{2}$. The vertex angles of regular polygons are not fixed, but shrink as the polygon grows; indeed, the deficit of vertex angles, compared with those of their Euclidean counterparts, scales linearly with the (hyperbolic) area of the polygon.

\subsection{Regular tilings}

The hyperbolic group contains a large variety of discrete subgroups. Among the most tractable examples are the so-called kaleidoscopic (or Coxeter) groups, generated by pure mirror reflections [18]. These groups are labelled here by their Conway orbifold symbol, $* a b \ldots$ [19]. (The * prefix indicates that the fundamental domain of the symmetry group is bounded by mirrors, and the digits following that prefix refer to the order of centres of rotational symmetry, $a b \ldots$ at vertices of the (polygonal) fundamental domain. The notation is explained in Ref. [19].) Coxeter's regular tessellations, or honeycombs, usually denoted $\{n, z\}$, are regular $z$-coordinated networks of polygons with $n$-sides, where the vertices of each polygon lie on cycles. Honeycombs are kaleidoscopes, with symmetries $* n z 2$. They can also be seen as symmetric arrangements of orthoschemes with all edges lying on mirrors of the pattern.

The edge length $a$ is determined by $n$ and $z$; the length required to form an $\{n, z\}$ honeycomb is given by hyperbolic trigonometry:

$$
a=2 \cosh ^{-1}\left(\frac{\cos \left(\frac{\pi}{n}\right)}{\sin \left(\frac{\pi}{z}\right)}\right) .
$$

According to hyperbolic geometry, the area of a single triangular orthoscheme is $\pi(1 / 2-1 / z-1 / n)$. The union of $2 z$ of those triangles form a (Voronoi) cell of the dual tiling $\{z, n\}$ surrounding each vertex; the area of this dual cell is the area of $H^{2}$ per vertex, the inverse of the density $\rho$ of vertices in the hyperbolic plane. It follows that

$$
\rho^{-1}=\pi\left(z-2-\frac{2 z}{n}\right) .
$$

Honeycombs $\{n, z\}$ are realisable in $H^{2}$ for all values of $n$ and $z$ satisfying $(n-2)(z-2)>4$. Consider 


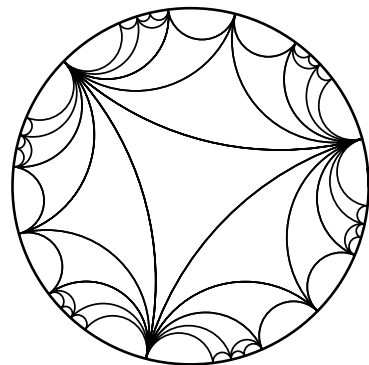

(a)

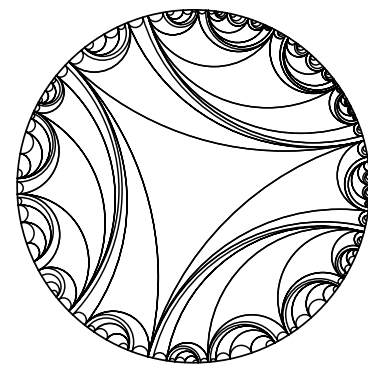

(b)
Fig. 2. Tilings of $H^{2}$ by triply asymptotic triangles (with vertex angles of zero) with kaleidoscopic $* 23 \infty$ (a) and 2223 (b) symmetries.

the compact surfaces $M$, built as quotients of $H^{2}$ by translation subgroups of $\{n, z\}$. The Euler-Poincaré characteristic $\chi(M)$, the number of vertices and the Gauss-Bonnet integral (integral of the Gaussian curvature $K$ over $M$ ) all scale linearly with the index of the subgroup, so that we can speak of an effective Euler characteristic per vertex (or fractional Euler characteristic) $\chi_{v}=\chi / V$. Indeed, Euler's Theorem asserts that the Euler-Poincaré characteristic of a surface $(\chi)$ is determined by the number of vertices $(V)$, edges $(E)$ and faces $(F)$ found in any network decorating the surface, $\chi=V-E+F$. Moreover, the $\{n, z\}$ tiling projected onto $M$ satisfies $2 E=z V=n F$, so that:

$$
\chi_{v}=1-\frac{z}{2}+\frac{z}{n} .
$$

Notice that $\rho^{-1}=-2 \pi \chi_{v}$ is consistent with the (global) Gauss-Bonnet Theorem and the constant value of the Gaussian curvature $K\left(H^{2}\right)=-1$.

By duality (replacing faces by vertices, and vice versa, as for $\{n, z\}$ and $\{z, n\}), \rho$ also describes the density of faces of the duals.

\subsection{Asymptotic tilings}

For fixed $n$, as the edge length $a$ grows, so does $z$. The angles between adjacent edges shrink until they eventually vanish. In this case, the edges become parallel and infinite (although the polygonal area remains finite). The entire hyperbolic plane can be tiled by copies of such "asymptotic" polygons. The most symmetric asymptotic triangular tiling is shown in Figure 2a. As all adjacent edges are parallel, the tiles can be translated relative to their neighbours an arbitrary length along their edges, so that a variety of other, less symmetric, tessellations are possible (e.g. Fig. 2b).

The duals of asymptotic tilings are trees; in particular the dual of the asymptotic $* 23 \infty$ triangular tiling is a Platonic three-coordinated Bethe lattice with equivalent edges and vertices (Fig. 3a).

The dual construction can also be extended to the less symmetric asymptotic tilings. In those cases, the dual consists of an infinite collection of disjoint trees, a "forest", discussed in more detail below (Sect. 3.2).

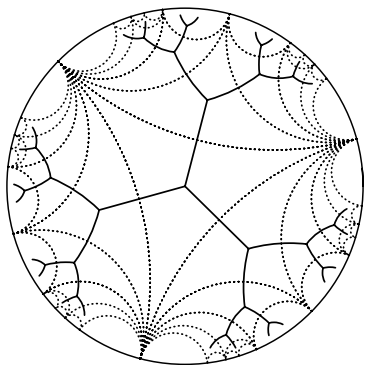

(a)

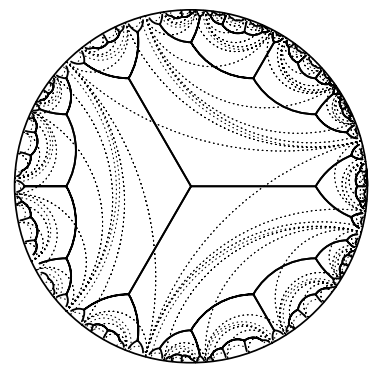

(b)
Fig. 3. (a) The Bethe lattice superimposed on its dual (the asymptotic tiling in Fig. 2a). (b) The dual of the asymptotic tiling of Figure $2 b$ consists of an infinite number of disjoint trees.

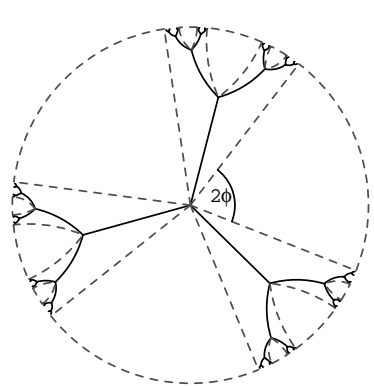

(a)

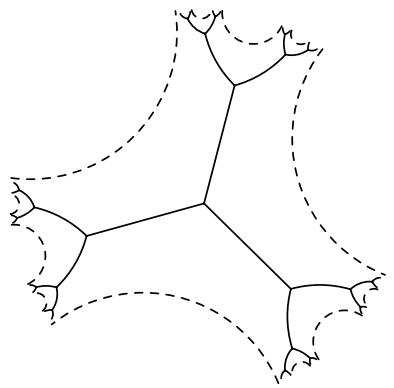

(b)
Fig. 4. (a) A supercritical three-connected tree in $H^{2}$, with some of the vacant sectors indicated (bounded by the dashed edges). (b) The convex hull of a tree (bounded by dotted geodesics).

\section{Forests in $\mathrm{H}^{2}$}

\subsection{Regular trees}

A Platonic, or "regular" tree is entirely characterised by its connectivity, $z=3,4, \ldots$ and edge length $a, a>a_{t}$, where $a_{t}>0$ is a threshold value. The tree is regularly embedded in $H^{2}$ with maximal symmetry: all edges are geodesic arcs of equal lengths and the angle between branches at each vertex is a multiple of $2 \pi / z$. We call it an $(a, z)$ tree, or $T(a, z)$.

The threshold value $a_{t}$ depends on $z$; its value is derived in Section 3.1.1. If edges are shrunk to $a<a_{t}$, the regular $z$-coordinated graph is generally self-intersecting in $H^{2}$ and its vertices densely fill $H^{2}$. For a countable set of values $a<a_{t}$ (Eq. 1), the loops close up to form regular polygons, yielding a hyperbolic $\{n, z\}$ honeycomb, as described in Section 2.3. For $a=a_{t}$, the vertices of $T(a, z)$ lie on horocycles, a marginal case that has been called a "critical tree" [21].

If $a>a_{t}$, we get a regular (supercritical) tree, whose vertices lie on hypercycles and are distributed inhomogeneously on $\mathrm{H}^{2}$ (Fig. 4), in contrast to the case of the critical tree (Fig. 3a). So embedded in $\mathrm{H}^{2}$, a tree divides the hyperbolic plane into several connected components, or vacant regions. Each of them is bounded by an open polygonal line, part of the tree. It contains radial sectors which are cones emanating from all vertices and spanning 
the same part of the horizon as their including region. By symmetry, they are all similar. For a $z$-coordinated tree, $z$ empty radial sectors emanate from every vertex (Fig. 4a). The angular divergence $2 \phi$ of the sectors depends only on the edge length $a\left(>a_{t}\right)$ and the connectivity $z$ of the supercritical tree:

$$
\cos (\phi)=\frac{\cos \left(\frac{\pi}{z}\right)}{\tanh \left(\frac{a}{2}\right)} .
$$

From elementary geometry, the convex hull of a tree may be defined in any of the following ways. i) As the smallest convex set containing the tree. ii) As the subset of $H^{2}$ spanned by all the (geodesic) lines joining two points of the tree. iii) As the complement of the union of the sub-domains common to all the sectors within every definite vacant region. That hull is an asymptotic polygon, bounded by an unlimited number of parallel geodesic edges (Fig. 4b). Following ii), above, the geodesics bounding the convex hull form the envelope of the geodesics linking all pairs of points in the tree. There is one bounding geodesic in every vacant region; it is the line that approaches the tree most closely among all the geodesics included in the same vacant region. This is discussed in more detail in Section 3.2.1.

\subsubsection{Tree group}

A regular tree $T$ is equivalent to a discrete hyperbolic group $G_{T}$, the stabiliser of $T$ in $H^{2}$, namely the subgroup of hyperbolic motions leaving the tree globally invariant. Depending on whether we consider $H^{2}$ as the complete hyperbolic group, or only as the group of proper (orientation preserving) hyperbolic motions, $G_{T}$ includes mirror reflections, or not.

The complete tree group is kaleidoscopic, and generated by three mirrors $A, B, C$. These generators are best described starting with a pair of edges $e_{1}, e_{2}$ forming an angle of $2 \pi / z$ at a shared vertex: mirror $A$ lies along $e_{1}$, mirror $B$ bisects the angle $\left(e_{1}, e_{2}\right)$ and mirror $C$ is on the perpendicular bisector of $e_{1}$. (Note that for odd values of $z$, mirror $B$ also contains a pointwise invariant edge) (Fig. 5).

The proper group $G_{T}$ is generated by a $z$-fold rotation $R=A B$ centred on a vertex and a 2 -fold rotation $S=A C$ centred at the midpoint of an edge ending at the same vertex.

The combinations $t_{k}=S R^{k}, k=1, \ldots, z-1$, generate the translation group $T_{T}$ of $T$; it is a subgroup of $G_{T}$ which turns out to be the free group generated by $t_{1}, \ldots, t_{z-1}$.

Written as a Moebius transformation [22], the product $S R$ corresponds to a matrix whose trace has magnitude:

$$
|\operatorname{trace}(S R)|=2 \cosh (a / 2) \sin (\pi / z) .
$$

The threshold value occurs when $S R$ is a horocyclic motion, that is when $|\operatorname{trace}(S R)|=2$, or

$$
\cosh \left(a_{t} / 2\right) \sin (\pi / z)=1 \text {. }
$$

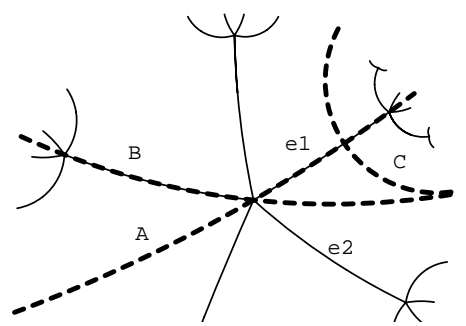

Fig. 5. Generators of the reflection group of arbitrary tree, here with $z=5$.

When $a>a_{t}(|\operatorname{trace}(S R)|>2)$, the product $t_{1}=R S$ is a genuine hyperbolic translation.

The orbit of a vertex containing the centre of rotation $R$ (together with the adjacent edge) under the cyclic subgroup $t_{1}^{n}, n=\ldots,-1,0,1, \ldots$ is one of the polygonal lines of $T$ (bounding a vacant region). The unique invariant geodesic of $t_{1}$ - the guiding geodesic of $t_{1}$ - is the bounding geodesic closest to the polygonal line. All the bounding geodesics of the tree, defining its convex hull, can be obtained similarly.

\subsection{Close packed forests}

We have seen that $H^{2}$ can be planted with an infinite collection of congruent trees, without overlaps, contacts or intersections. That is possible provided there are regions left vacant by a single $T(a, z)$, in which case the angular divergence of $T(a, z)$ given in equation (4) exceeds zero. Thus $\phi=0$ implies another expression for the critical edge length:

$$
a_{t}=2 \tanh ^{-1}(\cos (\pi / z)),
$$

equivalent to that of equation (5).

Clearly, the density of these $T(a, z)$ trees is maximised when the bounding geodesics of pairs of neighbouring trees coincide with each other, so that their convex hulls share a common edge and the whole set covers $H^{2}$ without overlaps. We call this arrangement a "close-packed" or "dense forest", $F(a, z)$ (mathematically speaking, our forests would only be "relatively dense" in $H^{2}$ ). This forest is indeed dense: when trapped in such a forest, there are only two escape directions: forward and backwards along the geodesic separating the pair of closest trees which surround you...

\subsubsection{Distance}

To quantify this notion of close-packing, we require a measure of distance. The distance between two points is the hyperbolic length of the (unique) geodesic arc joining those points. The distance between a point $x$ and a tree $T$ is the distance to the point $y$ on $T(a, z)$ closest to $x: d(x, T)=\min _{y \in T} d(x, y)$. One could similarly define a distance between trees as the minimum $d(x, y)$ over $\left\{x \in T_{1}, y \in T_{2}\right\}$, however, this is an inappropriate measure for evaluation of densities. So we choose a different convention. 


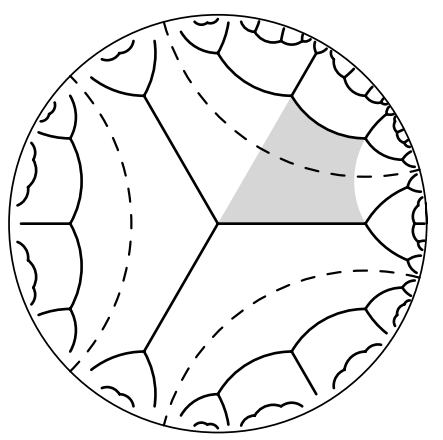

Fig. 6. A three-coordinated forest, showing three separating geodesics (dashed), and a single linking quadrilateral.

If the trees have non-empty intersection, we set $d\left(T_{1}, T_{2}\right)$ to zero. If the trees do not overlap, first notice that each of them is entirely contained in a vacant connected domain of the other (both being infinite, see Fig. 4). The polygonal boundary (in $T_{1}$ ) of the region containing $T_{2}$ is designated as the polygon $p_{1}$ of $T_{1}$ facing $T_{2}$ (or visible from $T_{2}$, as we also say); reciprocally, $T_{2}$ contains a polygon $p_{2}$ facing $T_{1}$. For any $x$ in the polygonal region enclosing $T_{2}$, the minimising $y \in T_{1}$ lies in the polygonal boundary $p_{1}$; in other words, $d\left(x, T_{1}\right)$ coincides with the distance to the polygonal line $p_{1}$. We define the distance between two trees as the average of $d\left(x, T_{1}\right)$ over the polygon $p_{2}$ of $T_{2}$ facing $T_{1}$ :

$$
d\left(T_{1}, T_{2}\right)=\lim _{L \rightarrow \infty} \frac{1}{L} \int_{p_{2} \cap B_{L}} d\left(x, T_{1}\right) \mathrm{d} x,
$$

where $B_{L}$ denotes a hyperbolic disc of diameter $L$.

When the trees belong to a regular close packed forest, the mutually facing polygons are parallel, in the sense that the distance $d\left(x, T_{1}\right)$, is uniformly bounded as $x$ runs along $p_{2}$; in fact, it is a periodic function of the abscissa $x$ along $p_{2}$. Define a linking quadrilateral $(q)$ as a quadrilateral obtained by joining a pair of neighbour vertices of $p_{1}$ to a pair in $p_{2}$ by geodesic arcs, the two pairs facing each other (Fig. 6). Since both pairs are separated by an edge of length $a$, and successive vertices lie at the same distance from their partners in the other tree, the quadrilateral $q$ is a parallelogram (Appendix A). By periodicity, the distance $d\left(x, T_{1}\right)$ is proportional to the area of a joining quadrilateral $q$ divided by the edge length $a$.

\subsubsection{Density}

Given a close-packed forest, $F(a, z)$, linking quadrilaterals $q$, introduced in the previous section, can be defined between all pairs of mutually facing edges. Globally, this defines a tiling of $H^{2}$ by quadrilaterals, with $2 z$ tiles meeting at every vertex. This tiling resembles the regular tessellation, $\{4,2 z\}$, though the tiles are parallelograms rather than regular squares.

The density of the forest, $\rho$, is the average number of vertices per unit area. For a close-packed forest of regular trees, $T(a, z)$, it is equal to the density of vertices of the tiling by $q$ 's, since both sets of vertices coincide, viz.

$$
\rho=\frac{4}{2 z \operatorname{area}(q)} .
$$

It turns out that there are a multitude of distinct regular close-packed forests. Any part of the forest, lying on one side of a bounding geodesic, can be translated relative the rest (on the other side of the geodesic) by an arbitrary translation along this geodesic. This gives regular forests an infinite number of degrees of freedom. However, such translations do not change the inter trees distance. Further, a stronger result holds: all regular close-packed forests of common tree coordination, $z$, have equal density, and the maximal density of a (non overlapping) $F(a, z)$ forest is independent of $a$.

A proof of these two statements is postponed to the Appendix.

Given these results, we are free to shift the trees along separating geodesics so as to bring symmetry operations of neighbouring trees to coincidence without changing the density of the tree packing. For example, shift $T_{2}$ until an $A$ mirror of $T_{2}$ (transversal to the guiding geodesics) coincides with an $A$ (or $B$ or $C$ ) mirror of $T_{1}$. The regularity of the trees implies a set of coincidences, sufficient to enlarge the symmetry group of the pattern to a full discrete subgroup $G_{F}$ of $H^{2}$, with compact fundamental domain. The guiding geodesics (already supporting translational symmetries of neighbouring trees) may then also become mirrors or glide reflections. In particular, we are free to choose the particular geometric setting in which the quadrilaterals $q$ are regular hyperbolic squares, so that the symmetry group of the forest becomes that of the honeycomb group, $* 24(2 z)$. Thus the density of any close-packed forest $F(z)$ is:

$$
\rho=\frac{1}{\pi(z-2)}
$$

as follows at once from equation (2).

\subsection{Examples}

\subsubsection{Three-coordinated forests, $z=3$}

The kaleidoscopic group relevant to the $P / D / G$ surfaces is $* 246$, (to be discussed in Sect. 4.2 ), characteristic of the $\{4,6\}$ honeycomb [23]. We derive forests commensurate with this example first. Recall the parametrisation of the tree by its edge length $a$. It follows from equations $(5,6)$ that the shortest edge length that leads to a supercritical tree commensurate with $\{4,6\}$ is that of an edge of the regular 4-gon formed under the orbit of the $2 * 32$ symmetry group, namely $a=\cosh ^{-1}(3)$. The orbit of the edge under this group forms a close-packed forest, shown in Figure $7 \mathrm{a}$. The regular 4 -gon of $\{4,6\}$ plays the role of a linking quadrilateral (Sect. 3.2.1) for this forest of densely packed $(a, 3)$-trees. Moreover the set of tree nodes coincides with the set of 6 -fold vertices of the tiling. It follows 


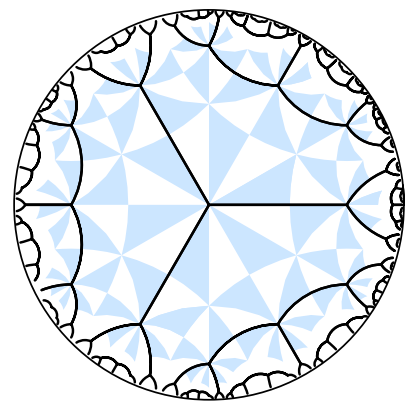

(a)

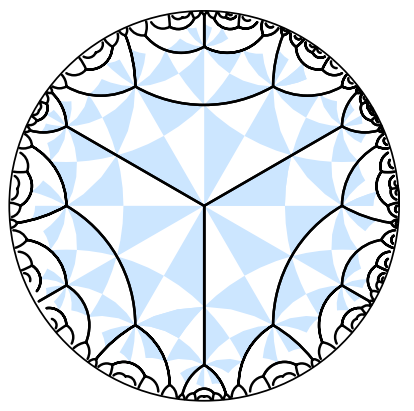

(b)
Fig. 7. Forests of three-coordinated trees of symmetry $2 * 23$, superimposed on the $\{4,6\}$ tiling. (a) Edges along an edge of the $\{4,6\}$ tiling (length $\left.\cosh ^{-1}(3)\right)$ and (b) edges along a square diagonal (length $\left.\cosh ^{-1}(5)\right)$.

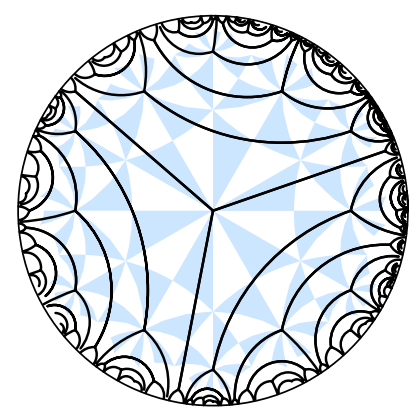

(a)

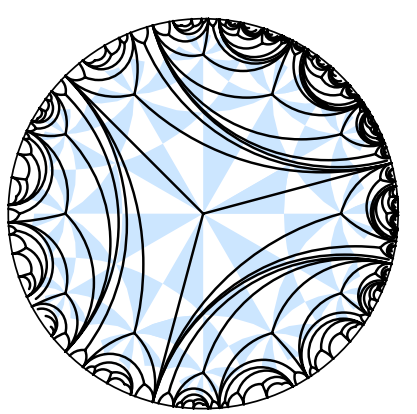

(b)
Fig. 8. Two forests of symmetry 2323. (a) Three-coordinated forest with all edges equal to a double square diagonal, $\cosh ^{-1}(15)$. (b) Limit case forest of three-fold trees in $H^{2}$. Each "tree" is made of just three lines meeting at a single vertex, and is a three-fold star.

from equation 8 that the density of this forest - whence of any close-packed three-connected forest - is $\pi^{-1}$.

A sequence of forests can be generated that are commensurate with the $\{4,6\}$ honeycomb, with successively longer edge lengths, $a$ (Figs. 7b, 8a). A slightly less divergent tree results if a diagonal of the regular 4 -gon is used $\left(a=\cosh ^{-1}(5)\right)$, also of symmetry $2 * 32$ (Fig. 7b). Further members in the sequence can be formed by "leapfrogging" from previous members, using the rule that emerges from the first pair. All members whose edge lengths exceed $\cosh ^{-1}(5)$ have slightly lower symmetry than the first pair of forests, viz. 2322. (This is also the relevant symmetry for the first two members, if we restrict elements to proper operations). The generators of this group are $R, S, S_{1}, S_{2}$ where $R, S$ are the symmetry generators of the first tree (described in Sect. 3.1.1) and $S_{1}, S_{2}$ are two-fold rotations located at the centres of two 4 -gons sharing a common edge.

The limiting case of this sequence (initiated by Figs. 7 , 8a) differs from finite members, in that each tree degenerates to a star-like graph, with a single vertex, and three infinite edges (Fig. 8b).

There is an interesting connection between the first and limit members of this sequence of forests. The edges of

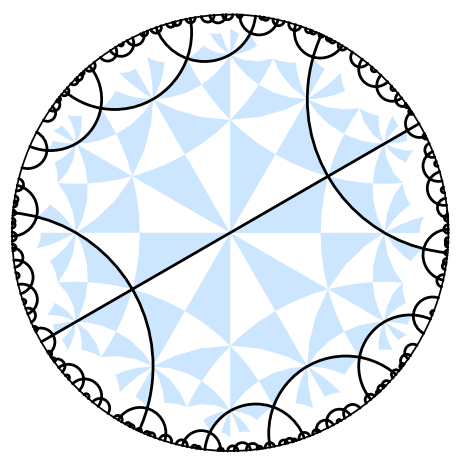

Fig. 9. Four-fold forest of symmetry $* 2224$, with edges of length $\cosh ^{-1}(5)$ on $\{4,6\}$.

the stars of the latter accumulate around specific geodesics which are bounding geodesics of the former (boundary lines of the convex hulls of the trees, Figs. 4b, 6). It is also worth noting that the convex hulls of the degenerate trees in the limit case are asymptotic triangles. The closepacked nature of the limit forest implies that the triangles tile $H^{2}$; this is precisely the tiling shown in Figure $2 \mathrm{~b}$.

\subsubsection{Four-coordinated forests}

Forests consisting of trees of connectivity four are readily built within the $\{4,6\}$ kaleidoscopic tiling characteristic of the $P, D, G$ family of surfaces. The density of these forests is a half that of the $z=3$ cases (cf. Eq. (8)), $\rho=$ $(2 \pi)^{-1}$. (An alternative derivation of the density follows from recognition that the parallelogram $q$ separating two trees contains 12 orthoschemes and $4 / 8=1 / 2$ vertices, implying an area per vertex equal to 24 orthoschemes.)

An infinite sequence of four-connected forests can be constructed, analogous to the three-connected case. The first member has edges equal to diagonals of the $\{6,4\}$ tiling. This forest, $F\left(\cosh ^{-1}(5), 4\right)$, with symmetry $* 2224$, is shown in Figure 9, with vertices at the centres of some of the regular 4-gons of the kaleidoscopic net. Higher order members of the sequence are generated by joining nextnearest neighbouring vertices, ...

\subsubsection{Six-coordinated forests}

In order to derive graphs on other TPMS, we choose decorations commensurate with symmetries other than the $* 246$ symmetry characteristic of the cubic $P, D$ and gyroid $G$ TPMS. Choose, for example, the $* 2226$ kaleidoscopic group, that is characteristic of the hexagonal $H$ TPMS. (cf. Sect. 4.2). A subset of the mirror lines of that group leads to a semi-regular $\{4,12\}$ tessellation of $H^{2}$ by hyperbolic parallelograms, with equivalent vertices, but two distinct edge lengths. Those 4-gons can be taken as the basis for a family of six-coordinated forests joining nearest vertices, diagonals, ...; as for the three- and four-connected forests. The simplest six-coordinated trees contain edges coincident with those of the 4 -gon that contain a $* 6$ junction of mirrors, and lengths exceeding the critical length 


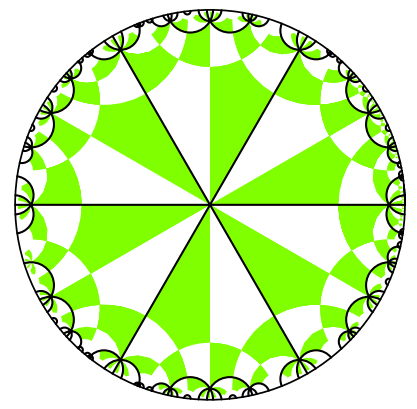

(a)

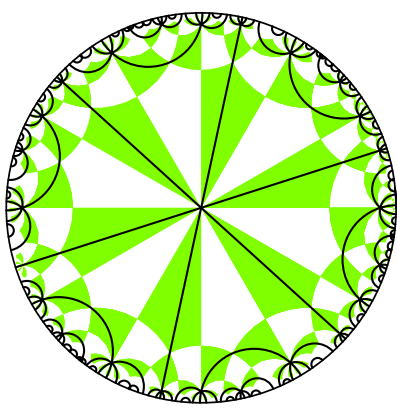

(b)
Fig. 10. Dense regular forests with symmetries (a) $* 2226$ and (b) 2226 , commensurate with the $* 2226$ kaleidoscopic group of the $H$ surface.

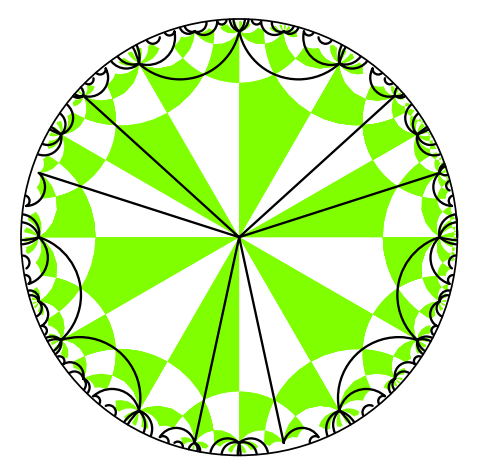

Fig. 11. A dense irregular forest commensurate with the kaleidoscopic tiling of the $H$ surface, of symmetry $22 * 3$.

for trees, $a_{t}$ (Eq. (6)). In general, two possible edges can be chosen, as the 4-gons are irregular. The resulting forests, of symmetry $* 2226$, illustrated in Figure 10 , are dense, with a vertex density of $(4 \pi)^{-1}$ (Eq. (8)). That is readily verified, noting that there is a one-to-one correspondence between vertices of the $\{4,12\}$ network, and invoking equation (2). The second member of the sequence, with edges along diagonals of the $\{4,12\}$ tiles, has symmetry 2226 (and is evidently also close-packed).

Other less symmetric six-coordinated trees can be superimposed on the kaleidoscopic tiling of the $H$ surface. For example, a forest of symmetry $22 * 3$ whose edges are the same length as in the 2226 forest, can be readily constructed, shown in Figure 11.

\section{Mapping forests to graphs on TPMS}

\subsection{Mapping $\mathrm{H}^{2}$ into $\mathrm{E}^{3}$ : coverings}

Our ultimate goal here is to derive examples of packings of disjoint regular graphs in $E^{3}$. So far, we have seen how to embed regular trees in a compact manner in $H^{2}$.

The idea is to use TPMS and the theory of coverings [24]. As $H^{2}$ is the universal covering of all the surfaces of genus $g>1$, there is a projection $p$ mapping the hyperbolic plane onto the surface $M$, in a way which is locally faithful (one-to-one) but globally several-to-one; each point $x$ in the surface $M$ is the projection of an infinite set of pre-images. This set, $p^{-1}(x)$, is the orbit of a single member $y_{0}$ under the fundamental group $\pi_{1}(M)$ of the surface lifted as a translation subgroup of $H^{2}$. (This is analogous to a lattice in Euclidean geometry; the plane $E^{2}$ is the universal covering of the torus $T^{2}$, or any surface of genus one, and the covering group is a $2 \mathrm{D}$ lattice.) Through the projection $p$, a pattern in $H^{2}$ will project into $M$ in a well defined way, provided this pattern is symmetric under the translations of $\pi_{1}(M)$.

Moreover, when dealing with periodic surfaces, such as the TPMS, we may require the final pattern in $M \subset E^{3}$ to have the same (translational) symmetry $L(M)$ as the bare surface. The lattice $L(M)$ can also be lifted to $H^{2}$, so that there is a translation group $T(M)$ representing both the loops $\pi_{1}(M)$ and the symmetries $L(M)$ of $M$. (Actually, $T(M)$ is the lift of the loop group of $M / L(M)$, a compact surface.)

\subsection{Kaleidoscopic groups}

The symmetry group of TPMS often contains symmetries in addition to translations. For example, the $P, D, G$ surfaces have cubic symmetry involving 24 proper rotations, or $48 O(3)$ operations belonging of the full kaleidoscopic group. Then the fundamental domain is a triangular orthoscheme which lifts, together with the point group, to an orthoscheme in $H^{2}[18,23]$. Through the projective map, and its (local) inverse, the universal cover of the TPMS now contains a representation of the point group as a hyperbolic kaleidoscopic group, that may be derived from the symmetry group of the Gauss map (whose range is in $S^{2}$ ) [25]. by "symmetry editing" [25], from $S^{2}$ to $H^{2}$ Note that the resulting symmetries in $H^{2}$ are precisely the in-surface symmetries of the TPMS. Of course, this does not imply that the graph has all the symmetries of the surface; in all the examples of Section 3.3, the forest is invariant under a subgroup strictly smaller than the symmetry group of $M$.

The tiling by orthoschemes offers a convenient reference frame to locate graphs in both $\mathrm{H}^{2}$ and the surface. This allows us to perform the mapping from $H^{2}$ to the surface $M$ even if, in general, we do not have an explicit formula for this mapping. (Note that $p$ can be made conformal if the surface is minimal.) In neighbourhoods of special points (e.g. vertices of the tiling, Wyckoff positions, or flat points of the surface), the mapping is easier to handle, because of symmetry or other properties, such as vanishing of curvature.

The algorithm to determine the relevant symmetry of the universal covering from that of the Gauss map of $M$ is simple for the surfaces we choose here: the locally isometric family of genus three cubic TPMS', the $P, D$ and $G$ (yroid) surfaces, the genus three (hexagonal) $H$ surface and the genus four (cubic) $I$-WP surface. The fundamental domains for the kaleidoscopic groups of the Gauss maps of those TPMS can be found in [26]. Those domains are spherical polygons, whose vertices are branch points of known order $b$ (possibly zero) [26]. The symmetry edits of the corresponding hyperbolic orbifolds are obtained as follows. Vertex angles of intersecting mirrors in $\mathrm{H}^{2}$ are 
Table 1. Kaleidoscopic symmetry groups characterising the Gauss maps of some simpler TPMS in $S^{2}$ (or, in the case of the $I$-WP surface, a triple covering of $S^{2}$ ), and corresponding symmetries of the representations of the TPMS in $H^{2}$, the surface genus per translational unit cell and space group symmetry in $E^{3}$.

\begin{tabular}{ccccc}
\hline \multicolumn{5}{c}{ Symmetry in } \\
Surface & $S^{2}$ & $H^{2}$ & $E^{3}$ & Genus \\
\hline$P$ & $* 243$ & $* 246$ & $\operatorname{Im} \overline{3} m$ & 3 \\
$D$ & $* 243$ & $* 246$ & $\operatorname{Pn} \overline{3} m$ & 3 \\
$G$ & $* 243$ & $* 246$ & $\operatorname{Ia} \overline{3} d$ & 3 \\
$H$ & $* 2223$ & $* 2226$ & $P 6_{3} / m m c$ & 3 \\
$I-W P$ & $* \frac{4}{3} 2 \frac{4}{3} 2$ & $* 4242$ & $\operatorname{Im} \overline{3} m$ & 4 \\
\hline
\end{tabular}

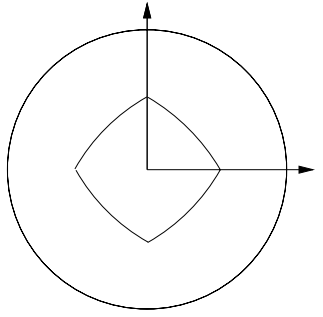

(a)

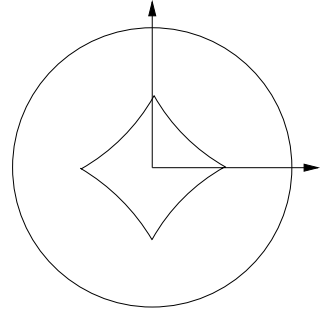

(b)
Fig. 12. Picture of a square patch enclosing 8 fundamental orthoschemes of the kaleidoscopic group for the $P, D$ and Gyroid surfaces, viewed (a) in $S^{2}$ by the Gauss map (or in $E^{2}$ by additional stereographic projection) and (b) in $H^{2}$ (with axes showing relative orientations).

shrunk by a factor of $\frac{1}{b+1}$ relative to those on $S^{2}$. (Genus three examples - the $\stackrel{b+1}{P} D, G$ and $H$ surfaces - have first order and the genus four example - the $I$ - WP surface - has second order branch points.) The relevant kaleidoscopic groups are listed in Table 1; the $P, D, G$ example is drawn in Figure 12. Note that the members of an isometry class, or Bonnet family, admit a common covering [27]. This is the case for the three TPMS $P, D$ and $G$ which are Bonnet transforms of each other; therefore the settings are common to all three.

For the $P, D, G$ examples, the kaleidoscopic group is $* 246$, that allows a kaleidoscopic net of $H^{2}$ containing only triangles, each with vertex angles of $\frac{\pi}{2}, \frac{\pi}{4}$ and $\frac{\pi}{6}$ (Coxeter's "orthoscheme" [28]). A regular subgraph of that tessellation is the regular $\{4,6\}$ honeycomb, originally used by Sadoc and Charvolin to characterise the $P, D$ and $G$ surfaces in $H^{2}$ [23].

The kaleidoscopic nets of the $H$ and $I$-WP surfaces are less regular, with symmetries $* 2226$ and $* 2424$ respectively. In both cases, the relevant fundamental domains are geodesic quadrilaterals, with vertex angles of $\frac{\pi}{2}, \frac{\pi}{2}, \frac{\pi}{2}$ and $\frac{\pi}{6}$ for the $H$ surface, and $\frac{\pi}{2}, \frac{\pi}{4}, \frac{\pi}{2}$ and $\frac{\pi}{4}$ for $I$ - $W P$ surface. Those angles alone are not sufficient to determine the shape of the quadrilaterals (in contrast to the rigidity of hyperbolic triangles with fixed vertex angles). The domain for the $I$ - WP surface consists of six adjoining hyperbolic triangles with vertex angles $\frac{\pi}{12}, \frac{\pi}{3}$ and $\frac{\pi}{2}$ (joined

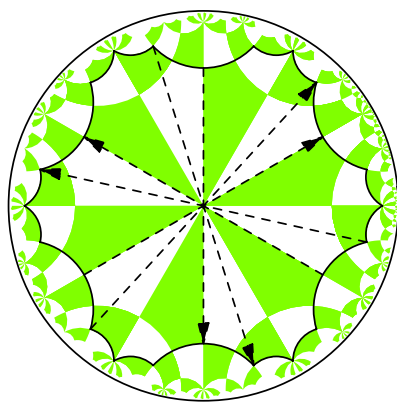

(a)

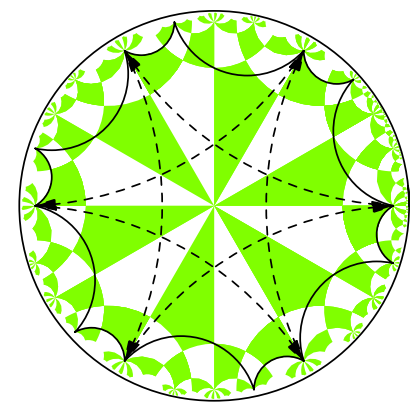

(b)
Fig. 13. (a) The translation group of $H$ lifted in $H^{2}$, together with a possible fundamental domain with 18 sides. (b) Another fundamental region (dodecagonal) for $T(H)$ and gluing vectors of $\pi_{1}(H)$; the dotted lines marked by arrows become closed loops in the projection mapping to surface $H$.

according to the scheme of Fig. 2a, Ref. [26]). The domain for the $H$ surface is a one-parameter family of quadrilaterals, parametrised, for example, by an internal angle, that defines the triangular decomposition of the quadrilateral (Fig. 13). That one-dimensional continuum of kaleidoscopic nets corresponds to the one-parameter family of $H$ surfaces, of variable ratio between their lattice parameters, $c / a$.

\section{3 $\mathrm{E}^{3}$ embeddings of forests: thickets}

We seek regular graphs in $E^{3}$, derived from coverings of the $P, D, G$ and $H$ surfaces. To maintain the regularity of the trees in $H^{2}$, we must ensure the scaling and placement of the trees is compatible with the covering; namely all vertices are located on identical points on the surface and all edges are located on identical trajectories on the TPMS. That implies that the trees in $H^{2}$ must be "commensurate" with the underlying kaleidoscopic group. In the examples to be seen, the forest group $G_{F}$ will be a subgroup of the kaleidoscopic group of the TPMS which contains the translation group $T(M)$, thereby fulfilling the conditions required by the covering map.

The mapping introduced above leads to a multiple covering of the TPMS. To generate the $E^{3}$ embeddings of forests, we force to identity the translations of the hyperbolic kaleidoscopic group belonging to $\pi_{1}(M)$. For example, in the case of $P, D, G$, the hyperbolic translation groups contain six generators which project to six lattice vectors in $R^{6}$. The loop groups of those 3 surfaces have a common subgroup which represents the loops of a surface embedded in $R^{6}$ and which is a covering common to $P, D, G$. Indeed, the $E^{3}$ embeddings of the TPMS can be considered as linear projections, to $E^{3}$, of the surface in $6 \mathrm{D}$ space (in fact, $C^{3}$ ) [27]. In each case, a set of three lattice vectors (whence a whole $3 \mathrm{D}$ sublattice) projects to 0 to form a Triply Periodic MS embedded in $E^{3}$.

Those relations define the gluing pattern of the hyperbolic domain, and are specific to the particular TPMS, distinguishing it from its isometric relatives. We consider only forests that share translational symmetries with their 
(a)

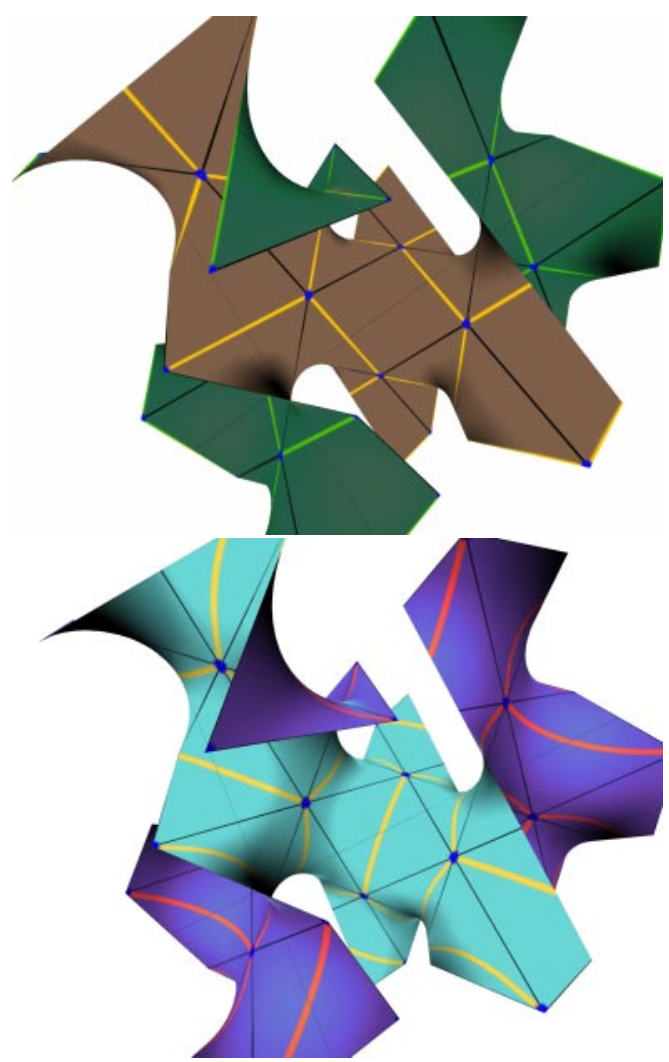

Fig. 14. Projection, onto the $D$ surface, of the sequence of forests commensurate with the $* 246$ orbifold group (characterising the $D / P / G$ surfaces): The forest in Figures $7 \mathrm{a}, \mathrm{b}$ map, respectively, onto (a), (b). The $\{4,6\}$ tiling is shown in black. The (yellow) edges of the thicket link vertices which are nearest-neighbours in (a), next-nearest neighbours in (b).

underlying surface (with one exception in Sect. 4.7.2), so that the analysis of global gluings can be confined to a single unit cell of the pattern.

The gluing patterns for the $P, D, G$ surfaces can be found in [23]. The gluings for the $H$ surface are shown in Figure 13b. These operations, required to form the TPMS, also act on the forest. The gluings induce noncontractible loops ("collar rings") on the surfaces, so that the embeddings of the ring-free hyperbolic forests in $E^{3}$ are 3D thickets, often containing entangled networks. The global structure of these thickets is discussed below.

The local form of projections of forests from $H^{2}$ to $E^{3}$ is illustrated by projections of the first four members of the sequence of forests illustrated in Figures 7, 8 onto a portion of the $D$ surface. The thicket edges shown in Figures 14, 15 are geodesics on the $D$ surface connecting vertices of the $\{4,6\}$ kaleidoscopic tiling (flat points on the surface).

We occasionally admit some geometric deformation of the thickets: curved edges are "rectified" with fixed endpoints, i.e. all vertex positions are frozen in $E^{3}$. Some examples are presented in the following sections. (c)

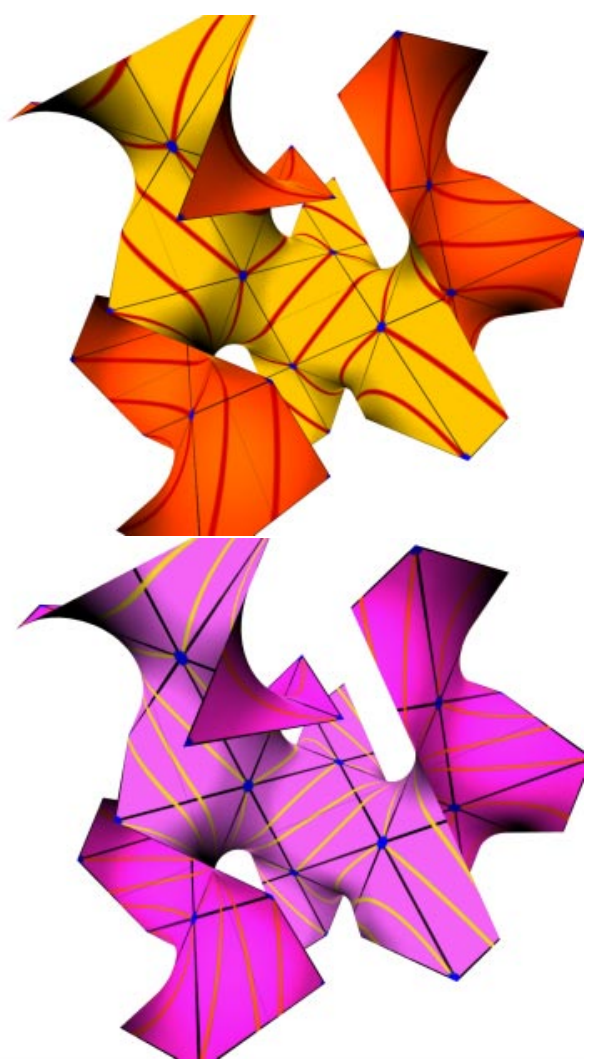

Fig. 15. Projection of forests onto $D$ (continued from Fig. 14). Network (c) is the map of Figure 8a. Thickets (c) and (d) contain edges of lengths $\cosh ^{-1}(15)$ and $\cosh ^{-1}(111.66 \ldots)$ respectively (length measured in $H^{2}$ ).
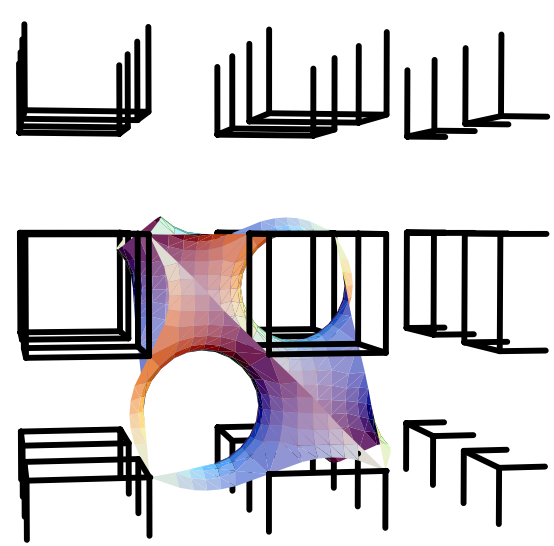

Fig. 16. The forest with $a=\cosh ^{-1}(3)$ - an edge of the regular $\{4,6\}$ tiling - folds on the $P$ surface to form an array of disconnected cubic clusters. The edges have been rectified.

\subsection{Forests on Schwarz' P surface}

4.4.1 $\mathrm{a}=\cosh ^{-1}(3)$

Consider first the sequence of forests on $\{4,6\}$, introduced in Section 3.3.1. The simplest member, with edges along an edge of the $\{4,6\}$ tiling, $F\left(\cosh ^{-1}(3), 3\right)$ (Fig. 7), folds on the $P$ surface to form a disconnected lattice of finite clusters, each of them equal to the set of edges 


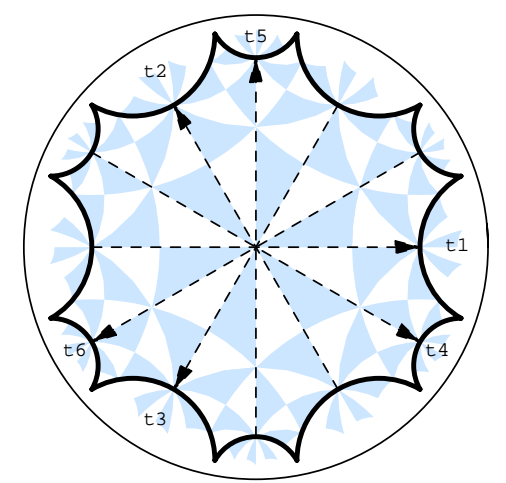

Fig. 17. Generators and dodecagonal fundamental domain of the translation group $T$ in $H^{2}$ for the $P, D, G$ minimal surfaces.

(and vertices) of an isolated cube (Fig. 16). The symmetry of the whole graph is simple cubic (SC), identical to that of the oriented $P$ surface itself.

We can take, as fundamental domain (for translations $L(P)=\mathrm{SC})$, the dodecagonal patch of the surface shown on Figure 16. This patch (conformally) lifts into the semiregular dodecagon of Figure 17 in $H^{2}$. The surface modulo $\mathrm{SC}$, in the 3D torus, is equivalent to the quotient of the hyperbolic plane by the (hyperbolic) translation group whose fundamental cell is the dodecagon $[23,27]$. Once the opposite edges of the dodecagonal region are properly identified, there remains only a single connected component in the graph (compare with Fig. 7a), which implies that there is one component (in this case, cubic cluster) per cubic unit cell. The side of the cluster is half of that of the fundamental cell.

\subsection{2 $\mathrm{a}=\cosh ^{-1}(5)$}

Next, set $a$ equal to the 4 -gon diagonal in $\{4,6\}$. This forest, $F\left(\cosh ^{-1}(5), 3\right)$ (Fig. $\left.7 \mathrm{~b}\right)$, folded on the $P$ surface yields a multiply-connected graph with eight connected components, all identical up to global translations (Fig. 20). All edges are face diagonals of the form $\left( \pm \frac{1}{2}, \pm \frac{1}{2}, 0\right)$.

The translation lattice of the graph $\bar{L}(P)$ is bodycentred cubic (BCC), corresponding to the translation group of the $P$ surface extended to include operations inverting the orientation of the surface. The $P$ surface is indeed a so-called balanced minimal surface [29] dividing space onto two congruent components (congruence being by translations of the type $\left.\frac{1}{2}(1,1,1)\right) . \bar{L}(P)$ contains the orientation preserving translations $L(P)=\mathrm{SC}$ as a subgroup of index two and the orientation reversing translation by $\frac{1}{2}(1,1,1)$. A convenient fundamental cell for $\bar{L}(P)$ is the dodecagon of Figure 18, with a half the area of the dodecagon introduced above. We take the cubic edge of $\bar{L}(P)$ as the unit length.

The translation lattice of each component is $2 \mathrm{BCC}$ (i.e. BCC with cubic edge of length two). Each component is identical to a single chiral labyrinth graph of the gyroid: the $(10,3)-a$ graph of Wells [1], also called $+Y^{*}$

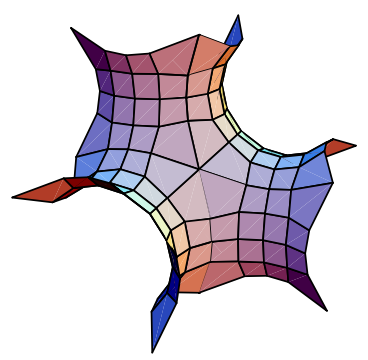

(a)

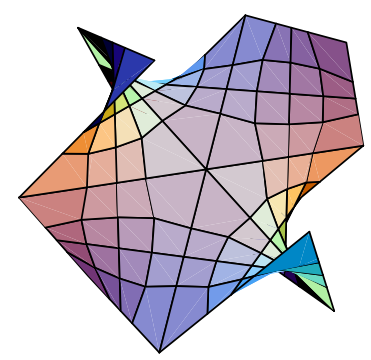

(b)
Fig. 18. Small dodecagonal fundamental piece of the extended translation group $\bar{L}$ for both the (a) $P$ and (b) $D$ surfaces.

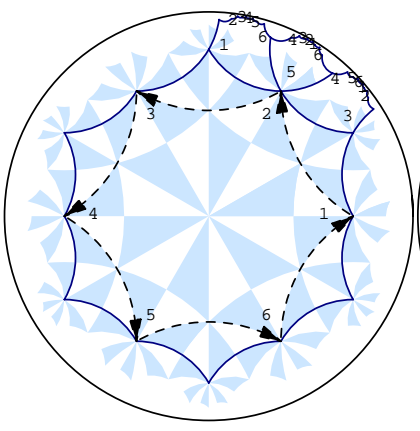

(a)

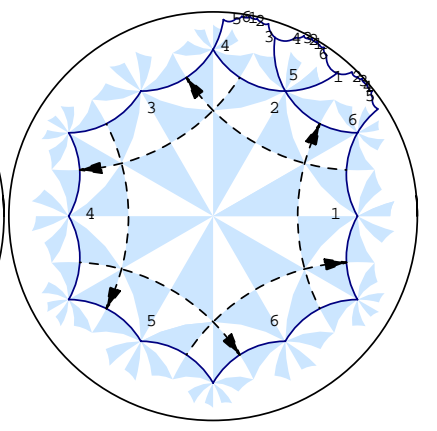

(b)
Fig. 19. Generators and small dodecagonal fundamental domain of the extended translation group in $H^{2}$ for the $P$ and $D$ surfaces. The numbers label some of the vertices of the 12 gon. (a) The generators of $\bar{T}(P)$ (for the $P$ surface) are reflections (through mirrors defined by the small edges of the orthoschemes of $\{6,4\}$ ) composed with translations along the arrows. (b) The arrows indicate glide reflections generating the group $\bar{T}(D)$ for the $D$ surface.

(right-handed) or $-Y^{*}$ (left-handed) [5]. When everything is reduced modulo $\bar{L}(P)$ (equivalent to projecting down to the non-oriented torus), there is just a single component. So, in $E^{3}$, the number of components is just the index of 2BCC in $\bar{L}(P)=\mathrm{BCC}$, which is indeed eight.

That the number of components modulo the lattice is one can be readily seen in $H^{2}$. Indeed, the semi-regular dodecagon (Fig. 19a) is again conformally related to a fundamental piece of the surface (Fig. 18a). The surface in the (non-oriented) torus is equivalent to the quotient of the hyperbolic plane by the (hyperbolic) glide reflections shown on Figure 19a. Once the opposite edges of the dodecagonal region are properly identified, one component remains in the graph. In principle, the symmetry lattice of the components (lift of 2BCC) could also be inferred by taking the quotient of the forest group by the fundamental group $\pi_{1}(P)$ of the $P$ surface. Then any residual translation of $P$ (mapping graph vertices onto graph vertices by construction) is a symmetry of the component provided the two vertices (origin and image) can be joined by a path within the graph. (Notice that the forest in the previous example, with shorter $a$, is not symmetric under the extended group $\bar{L}(P)$ of $P$.) 
(a)

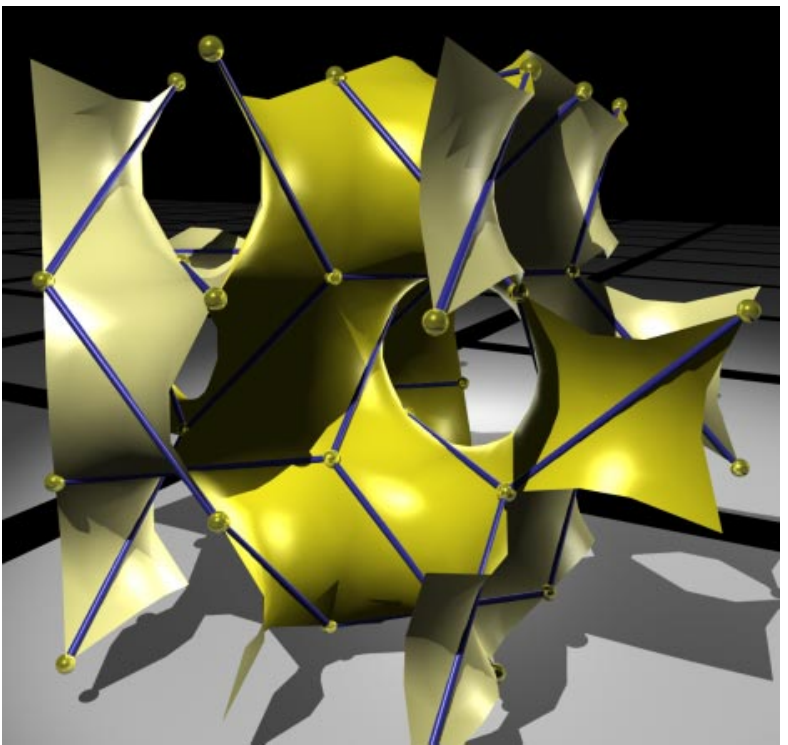

(b)

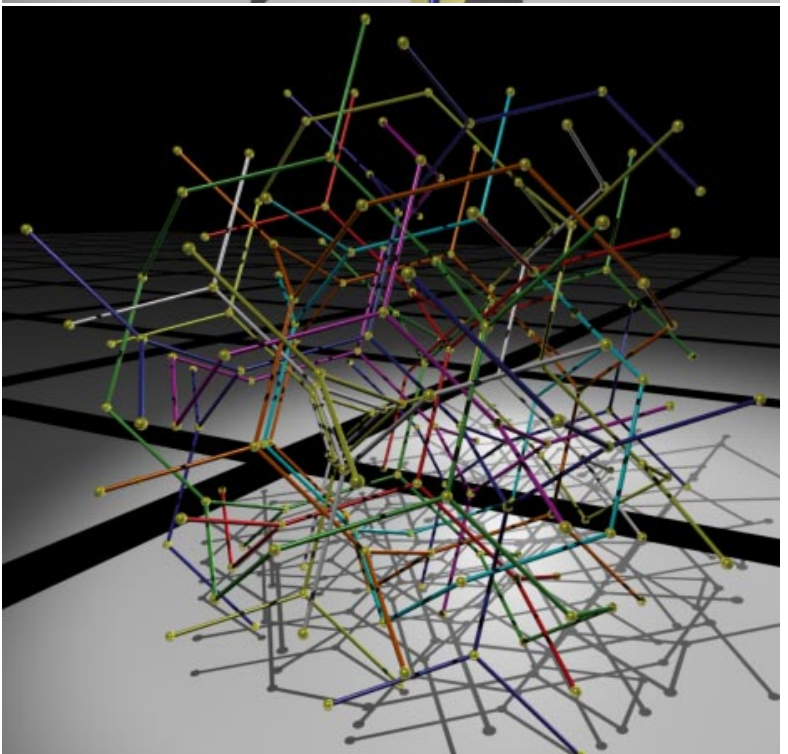

Fig. 20. (a) 3 -fold forest, with edge length $\cosh ^{-1}(5)$ on the $P$ surface. (b) The same graph, with its eight connected components coloured distinctly.

\subsubsection{Coordination $\mathrm{z}=4$}

The covering projection $p$ maps the four-coordinated forest of Figure 9 onto the $P$ surface to form a graph with a laminar structure, containing an infinite number of parallel connected components. It consists of a stack of regular $2 \mathrm{D}$ square grids (edge $=2^{-1 / 2}$ in the former units), with a spacing of $1 / 2$ between the layers.

\subsection{Forests on Schwarz' D surface}

$4.5 .1 \mathrm{a}=\cosh ^{-1}(3)$

The shortest forest, $F\left(\cosh ^{-1} 3,3\right)$, yields an entangled graph in $E^{3}$ consisting of four connected $+Y^{*}$ (or $-Y^{*}$ ) components, with 2BCC internal symmetry (Fig. 21). The presence of four components can be established as follows. (a)

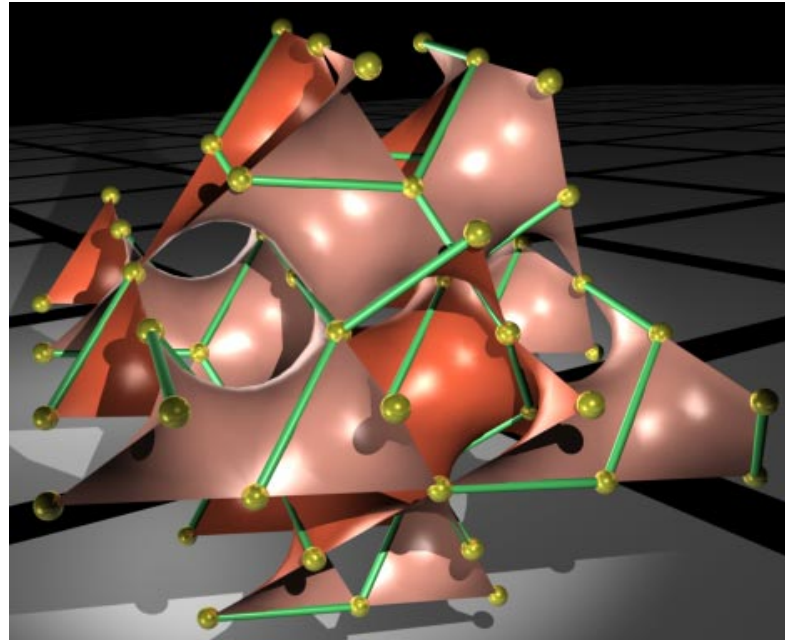

(b)

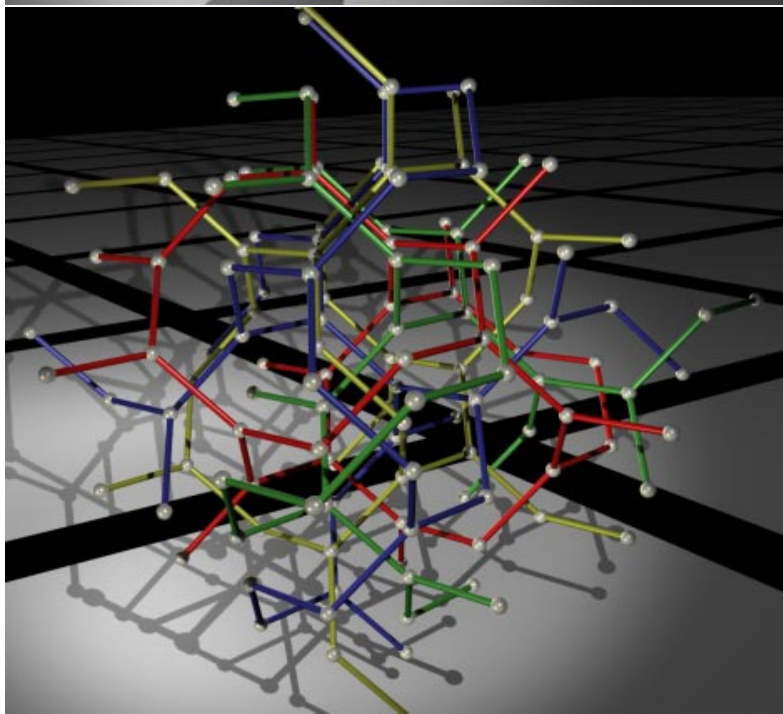

Fig. 21. (a) 3-fold forest, with edge length $\cosh ^{-1}(3)$ on the $D$ surface. (b) The same graph, with its four connected components coloured distinctly.

The translation symmetry of both the surface and the graph in $E^{3}$ is $\bar{L}(D)=\mathrm{SC}$. The SC translations do not preserve the orientation of the $D$ surface, therefore the surface modulo $\mathrm{SC}$ is a non-orientable surface in the torus $T^{3}$ (as for the $P$ surface). The orientation-preserving translations form a sublattice $L(D)=2 \mathrm{FCC}$ (face-centred cubic) of index two in $\bar{L}(D)$. The index of $2 \mathrm{BCC}$ in $\mathrm{SC}$ is four, corresponding to the four components of the graph. (Indeed, as can be checked in $E^{3}$, there is just one component in the graph modulo all the translations $\bar{L}(D))$.

Note that the orbits of each component under either $\mathrm{SC}$ or $2 \mathrm{FCC}$ yield the same pattern, namely the union of four $\left(\right.$ all + or all - ) $Y^{*}$ nets.

A possible fundamental patch of the surface for the (orientation breaking) SC lattice is the small dodecagon SDo (Fig. 18b). Its counterpart in $H^{2}$ is shown in Figure $19 \mathrm{~b}$, together with a set of lifted mirror-translations generating the extended translation group $\bar{T}(D)$. Compared with Figure 7, it appears that the forest modulo $\bar{T}(D)$ reduces to a single component in $H^{2} / \bar{T}(D)$, leaving 
only one component in $D / \bar{L}(D)$. (This would not be the case if the generators did not include (glide) reflections.)

$$
\text { 4.5.2 } \mathrm{a}=\cosh ^{-1}(5)
$$

The next 3-forest, with $a$ lying on diagonals of the regular 4-gons of the $\{4,6\}$ tiling of $H^{2}$, folds in the $D$ surface to form a lattice of disjoint $\{3,3\}$ tetrahedra (displayed in [30]). The tetrahedra enclose the nodes of only one of the labyrinths of $D$, a diamond structure. The lattice of translational symmetry is FCC.

\subsubsection{Coordination $z=4$}

Our last example of a forest on the $D$ surface is a wellknown bicontinuous structure. The 4-coordinated forest $F(a, 4)$, with $a=\cosh ^{-1}(5)$, folds on the $D$ surface to form a pair of identical entangled nets that are topologically equivalent to that of the diamond network (see [30]). The edges lie along principal directions of the TPMS, and are accordingly curved. We can straighten those edges in $E^{3}$ without moving graph vertices. The resulting regular graph describes the labyrinth structure of the $D$ surface itself.

\subsection{Forests on Schoen's G surface}

$$
\text { 4.6.1 } \mathrm{a}=\cosh ^{-1}(3)
$$

When folded on the $G$ surface, the forest $F\left(\cosh ^{-1}(3), 3\right)$ becomes an entangled subgraph of the regular diamond network, with two components, of the same chirality. All the graph edges point along (111) directions (Fig. 24). The symmetry group of the whole pattern is $L(G)=$ BCC, however, each of the two components has only SC translation symmetry ( $\mathrm{SC}$ is a sublattice of index two in $\mathrm{BCC}$ ). This is consistent with the fact that the two components are mapped onto each other by translations from $L(G)$, leaving only one component in $T^{3}$.

The covering of a fundamental patch of the $G$ surface is the dodecagon of Figure 17. (Only orientation-preserving translations need be considered since the $G$ surface is unbalanced, at least by pure translations, ruling out glide reflections). As already noticed, the forest $F\left(\cosh ^{-1}(3), 3\right)$ consists of one component, modulo this translation group. This confirms that there is only one component in $T^{3}$.

The coordination sequence [31-33] for either of the two components is identical to that of the $Y^{*}$ graph, supporting the contention that each component is a deformation of that network. The full graph thus consists of a pair of entangled (and distorted) $+Y^{*}$ or a pair of $-Y^{*}$ nets.

$$
\text { 4.6.2 } \mathrm{a}=\cosh ^{-1}(5)
$$

We turn next to the forest $F\left(\cosh ^{-1}(5), 3\right)$. Like the previous example, this folds in the $G$ surface to form a network subdivided into two connected components (Fig. 24c). The translational symmetry of the pattern is BCC, as is the symmetry of each component. The number of components modulo the BCC lattice is also two, in agreement with the

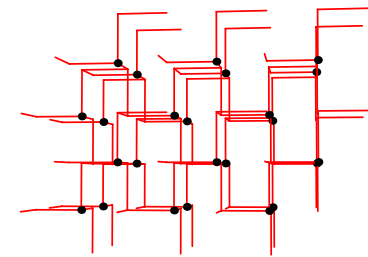

(a)

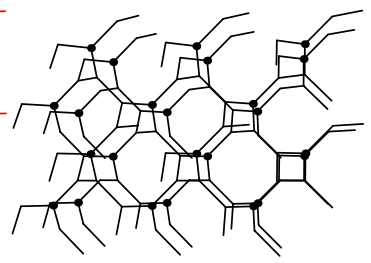

(b)
Fig. 22. (a) One of the two chiral components of the net in fig. 24(c) after straightening of edges in $E^{3}$. (b) A continuous deformation, preserving periodicity, transforms it into the $(+$ or -) $Y^{*}$ net. The dots, forming a BCC lattice, are fixed points.

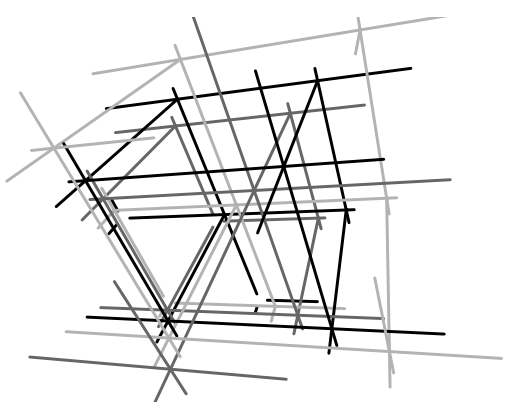

Fig. 23. The six-coordinated regular forest of Figure 10b folds on the $H$ surface to form a trio of identical rhombohedral graphs, each isomorphic to the six-coordinated simple cubic network.

result obtained in $H^{2}$ for the forest, modulo the lattice $T(P D G)$ (compare Figs. $7 \mathrm{~b}$ and 17$)$. The set of flat points on the $G$ surface forms a BCC lattice (with parameter half of the previous one). Since the unit cell of each component is a three-coordinated star, containing four vertices (and $9 / 2$ edges), only a half of the flat points are visited by a single component. This confirms that there are two components in the graph.

In this graph, all the edges (after straightening in $E^{3}$ ) are just cubic edges $\left(\frac{1}{2}, 0,0\right)$ (and equivalent ones under the cubic point group). Each component is, once again, a deformation of a chiral $Y^{*}$ net, (Fig. 22). An explicit possible interpolation scheme (isotopy, in mathematical parlance) is to let the bonds equal

$$
\left(e_{1}, . ., e_{6}\right)=\frac{1}{4}\left(\begin{array}{cccccc}
x & x-2 & 0 & 2-x & 0 & x \\
2-x & 0 & -x & 0 & -x & x-2 \\
0 & x & x-2 & x & 2-x & 0
\end{array}\right)
$$

with $x$ varying from zero (each component of the forest on $G$ ) to unity (the cubic $Y^{*}$ net). At all stages during the isotopy $(x \in[0,1])$, the graph retains its connectivity and its BCC translational symmetry (e.g. $e_{1}-e_{3}+e_{4}=$ $\left.\left(\frac{1}{2}, \frac{1}{2}, \frac{1}{2}\right)\right)$.

\subsection{Forests on Schwarz' H surface}

A unit cell of the $H$ surface is projected from $H^{2}$ as shown in Figure 13b (by identifying origin and end 


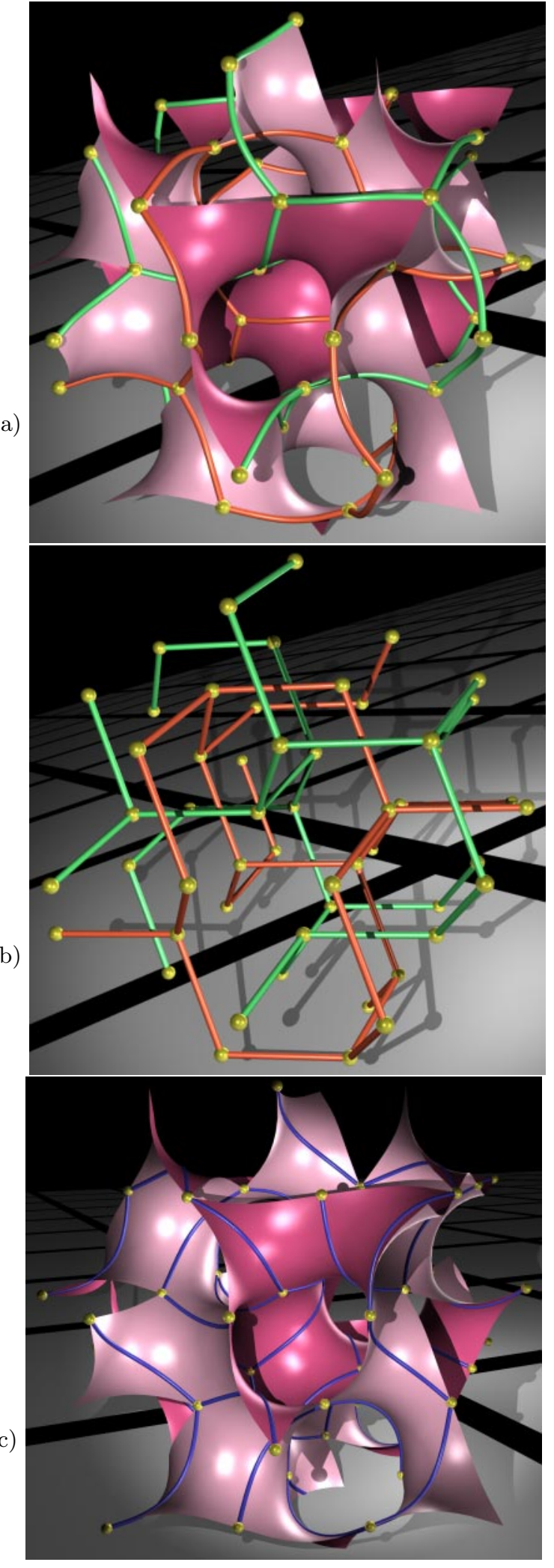

Fig. 24. (a) 3-fold forest, with edge length $\cosh ^{-1}(3)$ on the $G$ surface. (b) The resulting pair of distorted chiral $Y^{*}$ graphs, formed by straightening all edges while fixing vertices. (c) 3-fold forest, with edge length $\cosh ^{-1}(5)$ on the $G$ surface.

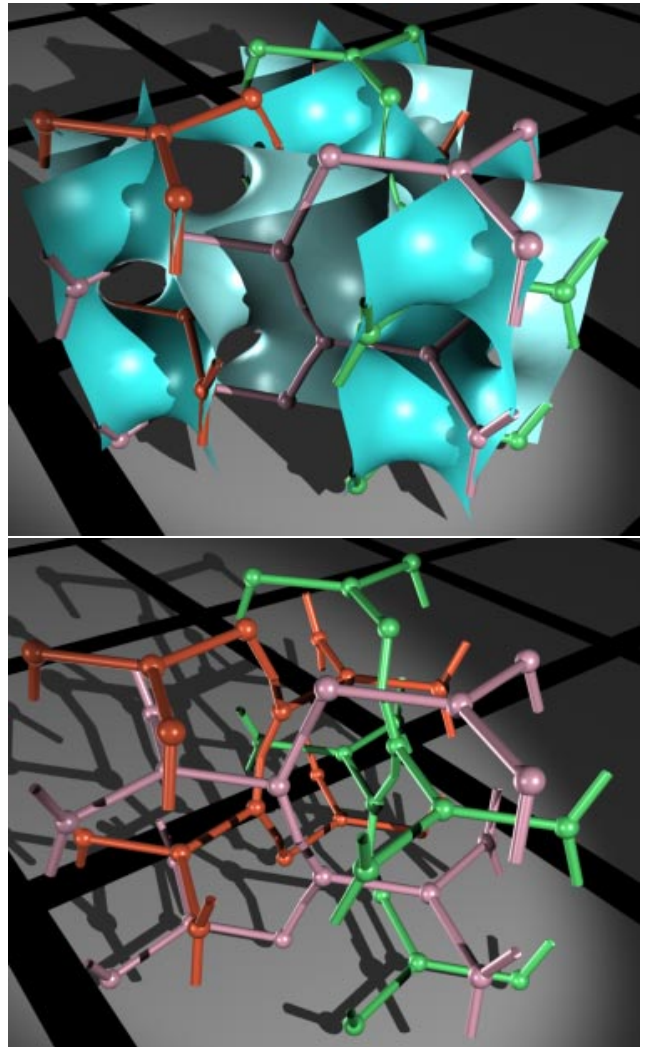

Fig. 25. Two views of a trio of three-coordinated nets in the $H$ surface generated from the transformation of Figure 27 applied to the forest shown in Figure 11.

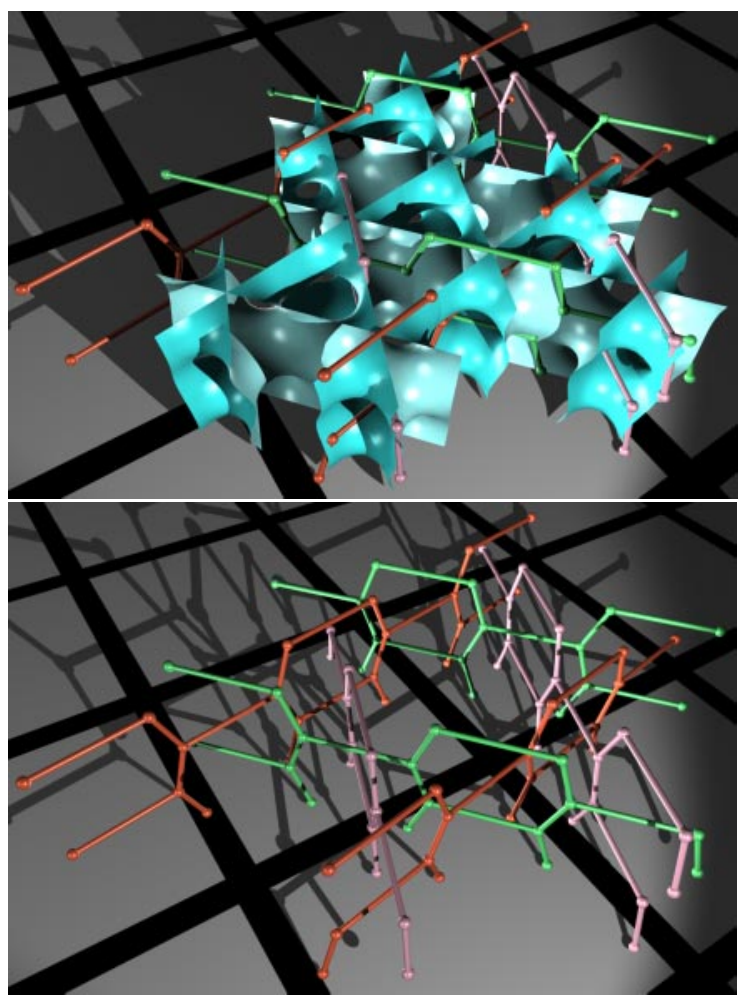

Fig. 26. Two views of the triple graphite network embedded in the $H$ surface. 
of the loop translations). Embeddings of forests derived from the irregular $\{4,12\}$ tessellation of $H^{2}$ lead to a variety of graphs, including novel three-component entangled networks. The simplest forests in the $z=6$ sequence of forests, with edges coincident with edges of the 4-gons (Fig. 10a), fold in the $H$ surface to form two different structures, corresponding to the pair of non-equivalent edges in the rectangular 4-gon ( $c f$. Sect. 3.3.3): one is a lamellar stack of planar $\{3,6\}$ networks, the other is a $2 \mathrm{D}$ lattice of infinite 6 -coordinated chains. Each component chain is a string of vertex-connected $\{2,3\}$ polyhedra.

The next member of the sequence contains edges spanning diagonals of the 4-gons (Fig. 10b). Three identical entangled components result in $E^{3}$, each a rhombohedral deformation of the six-coordinated simple cubic graph, Figure 23. (The latter is a single labyrinth of the $P$ surface.) In a way similar to the pair of labyrinths of $P$, related to each other by a translation along half a body diagonal, the three entangled components of the trio are mutually shifted by $1 / 3$ of a body diagonal along the ternary axis.

\subsubsection{An irregular forest on the $\mathrm{H}$ surface}

Another example of a trio of equivalent interwoven nets results from a distortion of the semi-regular six-coordinated forest shown in Figure 11. Each vertex is replaced by a symmetric three-armed star (Fig. 27), so that the coordination of all trees in the forest is reduced to three. Folding that irregular forest in the $H$-surface leads to a twoparameter family of graphs: one parameter is related to the axial ratio of the surface, and the other to the length of the arms in the stars. One member of that family is after straightening of the edges - a regular embedding in $E^{3}$, giving a trio of entangled three-coordinated $(8,3)-c$ graphs in $E^{3}[1]$, with vertex angles of $2 \pi / 3$, shown in Figure 25.

\subsubsection{Triple graphite}

Our last example is a pattern of entangled 2D graphs, composed of structures analogous to that described in Section 4.4.3. Each component is doubly periodic and planar, and the components are periodically stacked in directions normal to each plane. In contrast to the previous examples, this final example has three replicas of this layered pattern, disposed at 120 degrees to each other and mutually entangled to form an inter-growth of three graphitelike networks (Fig. 26).

To build such a structure in $E^{3}$, take a periodic stacking (in the $y$ direction) of hexagonal networks in the $x, z$ plane (either honeycombs or staggered edge-sharing quadrilaterals, as in brick walls). Place a vertical ternary axis (parallel to $O z$ ) at distance $a / \sqrt{12}$ from the plane of any component, and take the orbit of the laminar structure so that rotation around the axis induces a three-fold symmetry axis. None of the resulting components intersect
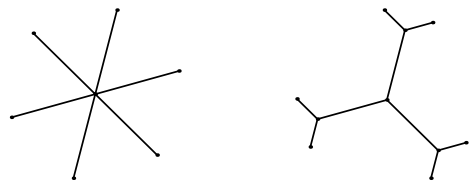

Fig. 27. Transformation of (left) a six-coordinated vertex into (right) four three-coordinated vertices.

Table 2. Summary of 3D thickets formed by decorating triply periodic minimal surfaces (TPMS) with forests of coordination $(z)$ three, four and six. The right-hand columns show the number and type of the component nets, derived from projections of the first two members of the sequence of forests (tree edges $a$ equal to, respectively, edges and diagonals of the 4-gons in the relevant $\{4, z\}$ tilings). $2 \mathrm{D}$ nets are labelled by their Schlaefli symbol, $\{n, z\}$. ("def." refers to geometric deformations of the graphs that reduce their symmetries, with no change in graph topology.)

\begin{tabular}{|c|c|cc|cc|}
\hline & TP & \multicolumn{2}{|c|}{ Forest 1: $a=$ edge } & \multicolumn{2}{|c|}{ Forest 2: $a=$ diag. } \\
$z$ & MS & $\#$ & component nets & $\#$ & component nets \\
\hline 3 & $P$ & $\infty$ & cubes $\{4,3\}$ & 8 & $Y^{*}$ (Fig. 20) \\
& $D$ & 4 & $Y^{*}$ (Fig. 21) & $\infty$ & tetrahedra $\{3,3\}$ \\
& $G$ & 2 & def. $Y^{*}$ (Fig. 24) & 2 & def. $Y^{*}$ (Fig. 22) \\
& $H$ & & 3 & def. (8,3)-c (Fig. 25) \\
& $H$ & & & $\infty$ & stacks of $\{6,3\}$ \\
& & & & & (Fig. 26) \\
\hline 4 & $P$ & $\infty$ & stacks of $\{4,4\}$ & & \\
& $D$ & 2 & diamond & & \\
\hline 6 & $H$ & $\infty$ & 6-strings, $\{2,6\}$ & 3 & rhomb. net (Fig. 23) \\
& $H$ & $\infty$ & stacks of $\{3,6\}$ & & \\
\hline
\end{tabular}

each other, and each 6-gon of one component is interlaced by an edge of another component.

As shown in Figure 26, this network can be embedded in the $H$ surface; then, it is a projection, into that surface, of a three-coordinated forest. Notice however that the symmetry group of the periodic net is lower than that of the naked surface. Indeed, in the horizontal $x, y$ plane, the generators of the lattice of the net are twice larger than those of surface $H$. As a consequence, to lift the pattern in the universal covering of $H$, one needs to consider a translational domain made of, at least, four fundamental cells of the $H$ domains (Fig. 13).

\section{Thickets in condensed matter - discussion}

A variety of novel thickets, sometimes entangled or interpenetrating, have been generated by projection into $E^{3}$ of regular forests in $H^{2}$. The connectivity and dimensionality of these thickets ranges from entangled 3-periodic crystalline graphs, layered stackings of 2-periodic nets, $2 \mathrm{D}$ lattices of 1-periodic graphs and lattices of finite graphs. We summarise the graphs generated in Table 2 . 
The aim of this work was to look for generalisations of bicontinuous topologies - characterised by a pair of interwoven 3D nets - to generic multicontinuous structures. One characteristic of such entangled structures is "maximal interpenetration" of interwoven nets, in the sense of Wells [1], with all rings punctured by a graph edge. Our article deals exclusively with forests of close-packed trees in $H^{2}$. This restriction is not unreasonable. Were the graphs to contain finite rings in $H^{2}$, those rings would appear in $E^{3}$ as (contractible) loops, bounding a patch of the embedding surface. But such rings cannot be threaded by edges of the same (or any other) graph in $E^{3}$. In other words, contractible loops are not compatible with maximal interpenetration, or entanglement, and regular entangled thickets must derive from projections of arrays of hyperbolic trees.

A further convenient reason for starting with regular trees is to allow formation of very symmetric thickets in $E^{3}$. Since regular finite-ring-size graphs in $H^{2}$ necessarily cover $H^{2}$ uniformly and are connected, the resulting graphs in $E^{3}$ contain only a single component [20]. Disconnected graphs with loops can be constructed in $\mathrm{H}^{2}$, but these are necessarily irregular, and require more complicated motifs with symmetrically distinct vertices.

The emergence of chirality in many of these graphs is an interesting feature, that is related to the mapping from $H^{2}$ to the TPMS. The choice of enantiomer depends on the direction of tilt of the forest edges with respect to the "gluing vectors" (involved in the map from $H^{2}$ into $\left.E^{3}\right)$. If that tilt vanishes, the resulting graphs are achiral, and chiral otherwise. (Notice that it seems impossible to embed trees, without crossings of edges, in $H^{2}$ that would yield both left-handed and right-handed patterns in $E^{3}$, see Sect. 5.2.) The technique thus offers a convenient recipe for generating a variety of chiral graphs in $E^{3}$. Such structures are of fundamental and commercial interest, given the need to synthesise chemical sieves capable of resolving racemic mixtures in to their enantiomeric components. We know of no other route to generation of such a wealth of chiral graphs.

Evidently, there remain an infinite number of other possible constructions. We have derived only embeddings in $E^{3}$ of the lower order forests, whose edges link closely neighbouring vertices of the kaleidoscopic tilings characteristic of the TPMS. In addition to the higher order members of the families of forests described above, we have not considered more highly-coordinated forests. Further, our analysis has been confined to the simpler genus-three TPMS, though the technique is readily generalisable to higher genus surfaces. We note, however, that the examples presented here are the simplest regular thickets, of coordination three, four and six.

\subsection{Liquid crystals}

It remains to discuss the possible relevance of these structures to condensed chemical systems. It is now well established that triply-periodic hyperbolic surfaces, specially the $P, D$ and $G$ TPMS, describe well the structure of surfactant and lipid bilayers formed in amphiphile-water mixtures, and the arrangement of microdomains in copolymer melts (for an overview, see [34]). These so-called bicontinuous phases contain two maximally inter-penetrating entangled network, or thickets, defined by the pair of labyrinth graphs of the TPMS. The thicket defining the $D$ surface topology has been derived in Section 4.5.3. The $P$ surface thicket is readily derived as a projection onto the surface of the regular $* 2626$ forest, $F\left(2 \cosh ^{-1}(3), 6\right)$. (The $G$ thicket, with an enantiomeric pair of components, is a projection of a forest with overlapping edges.) A number of other regular entangled structures, that are maximally interpenetrating, also emerge from our constructions.

The pair of identical enantiomers of distorted $Y^{*}$ graphs in the $G$ surface (Sect. 4.6) offers a novel chiral bicontinuous morphology. Similarly, the maximally interpenetrating graphs consisting of four and eight identical enantiomers of $Y^{*}$ graphs on the $D$ and $P$ surfaces are candidates for chiral quadra- and octa-continuous morphologies respectively. The possibility of such mixtures forming chiral meso-structures is an interesting one, that deserves serious attention.

Two novel (achiral) tricontinuous morphologies have also been generated by projections of six- and three-coordinated forests onto the hexagonal $H$ surface (Sect. 4.7). One is an attractive generalisation of the bicontinuous $P$ morphology, leading to three sixcoordinated component nets. The other has no bicontinuous analogue: it consists of three entangled hexagonal $(8,3)-c$ nets (using the notation of Wells, [1]). These offer promising candidates for novel mesophases.

In fact, this search for entangled nets was motivated by reports of novel morphologies in molten condensed "star" or "mikto-arm" copolymers, consisting of three immiscible polymer blocks grafted to a common centre [35-37]. These molecules are known to assemble into three distinct micro-domains, driven by the enthalpic constraint of micro-phase separation. Clearly, the two three-component thickets described here offer feasible structural models (with appropriate tuning of the volume fractions within each continuous domain). It is worth noting that according to transmission electron micrographs, the symmetry of these three-arm copolymer assemblies is most likely rhombohedral or hexagonal, though the reported structure of Sioula et al. [37], based on electron micrographs, differs from our tricontinuous ones, and is, in our opinion, less attractive than the models derived here.

\subsection{A complex molecular crystal: overlapping forests and $3 \mathrm{D}$ thickets}

These entangled thickets also offer novel models for crystals containing multiple frameworks, found, for example, in molecular crystals [38]. Indeed, the thicket consisting of four interwoven identical enantiomers of $Y^{*}$ on the $D$ surface (Sect. 4.5) has been found recently in a metallo-organic molecular crystal [39]. The molecular 


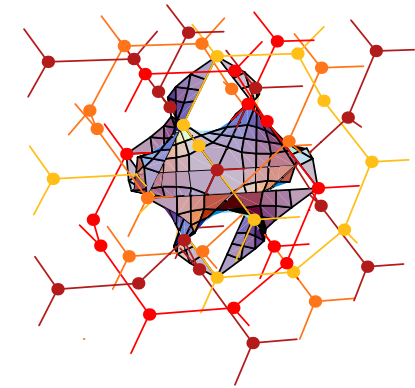

(a)

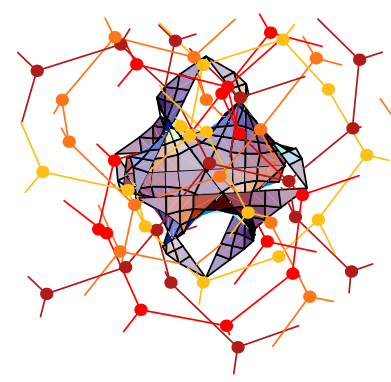

(b)
Fig. 28. (a) The right handed part of the Melbourne structure embedded in $D$. (b) Slight deformation of (a), produced by moving the vertices away from the surface along surface normals.

crystal discussed here is indicative of the possible importance of these periodic thickets to condensed materials.

Abrahams et al. [40] have reported a fascinating example related to this structure in the (organo-metallic) solid state, consisting of eight interwoven networks (that we call here the Melbourne structure). That structure too can be derived from forests in $H^{2}$, provided we extend somewhat our algorithm. So far we have reticulated $H^{2}$ with a single net, we now start with a pair of overlapping nets on the surface, then split them apart by pulling the nodes in directions normal to the surface.

Each of the eight components of the Melbourne structure are, to a first approximation, $Y^{*}$ graphs. A half of the components are left-handed, the remaining half righthanded. The first group of four (Fig. 2 in [40]) exactly coincides with the network described in Section 4.5.1 as a regular three-coordinated net embedded in the $D$ surface. The same method generates a right-handed net: it suffices to take the image of Figure 7a under a centre of inversion located at any of the vertices of the forest. When folded in the $D$ surface, this forest provides a second group of four three-coordinated networks, enantiomorphic to the first.

At this stage the vertices of the two groups coincide in $E^{3}$ (on the $D$ surface), as they do in $H^{2}$. We next remove this coincidence, forming eight disconnected nets with four left-handed and four right-handed components. The surface normal provides a naturally balanced direction along which to translate vertices (Fig. 28). More precisely, impose an orientation on the surface; this specifies a sign to the normal vector everywhere on the surface in a manner preserving continuity along the surface. Then shift the vertices of, say, the left-handed group along the positive normal and those of the right-handed group in the opposite (negative) direction, both by the same amount $x$. This, of course, induces some distortion of the graphs (clearly visible in Fig. 4 of [40]), but all the vertices are now distinct and separated by a distance at least $2 x$ (for some small $x$ ) (Figs. 29 and 30). This is precisely the Melbourne structure [40].

STH thanks CO and Cergy-Pontoise University for hospitality and financial support. $\mathrm{CO}$ thanks STH and Australian Na-

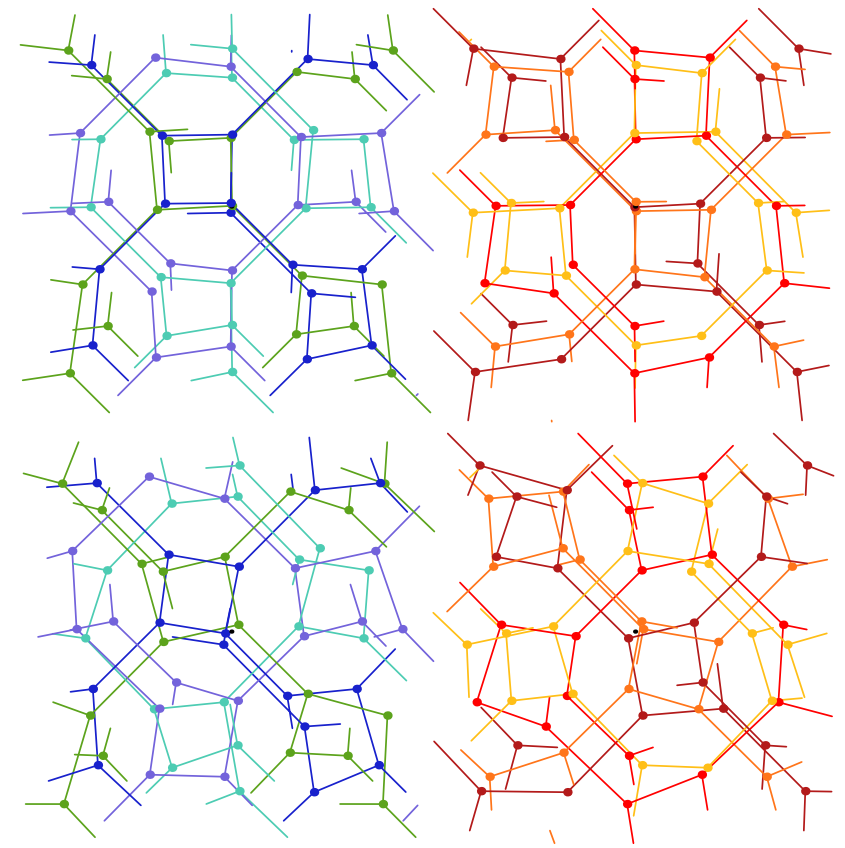

Fig. 29. Left- and right-handed parts of the Melbourne structure seen along a 4 -fold axis. Top: the net as it lies in the $D$ surface. Bottom: the deformed net, forming left- and righthanded components of the Melbourne structure.

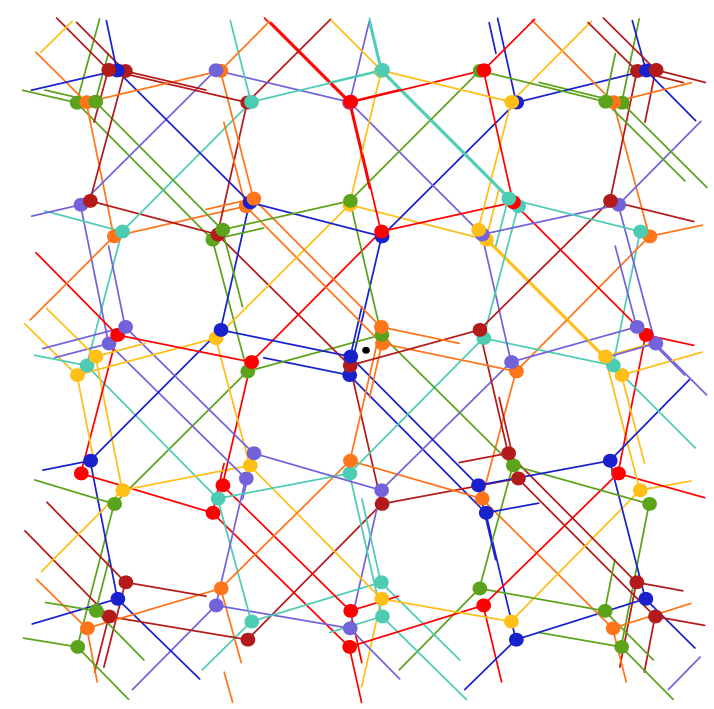

Fig. 30. Template for the Melbourne structure.

tional University for the same. Both authors thank Stuart Ramsden (Applied Maths, Canberra) for generating some of the figures and discussions.

\section{Appendix A}

A few facts on the geometry of close-packed forests of regular trees are proven here. The notation is the same as in Section 3.2.1. Let $g$ be the geodesic separating two nearest neighbour trees. Recall that $g$ is the guiding geodesic of $t_{1}$. 


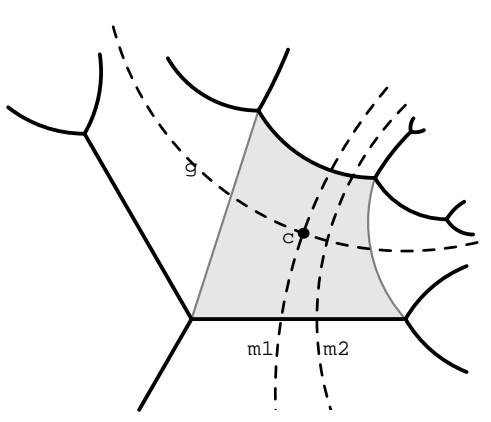

Fig. 31. A quadrilateral joining two trees and the mirror lines (dashed) entering the decomposition of the motion mapping one tree onto the other.

A1 The area of the linking quadrilateral $q$ is invariant when one tree is shifted with respect to the other by any translation along the guiding geodesic.

A2 The area of the linking quadrilateral $q$ is the same for all regular close-packed forests $F(a, z)$ (a varying, $z$ fixed).

\section{A.1 Independent of shift}

By construction, the polygonal line $p_{1}$ is periodic, that is invariant under the translations $t_{1}^{k}, k= \pm 1, \pm 2, \ldots$ The same is true for $p_{2}$, the polygonal line part of $T_{2}$.

Moreover, the tree $T_{2}$ is the image of $T_{1}$ by a glide reflection along $g$ and the same operation maps the polygonal line $p_{1}$ onto $p_{2}$. Now the glide reflection can be decomposed into a product of three reflections, $m_{g} m_{1} m_{2}$, where $m_{1}$ and $m_{2}$ are reflections through mirrors both perpendicular to $g ; m_{2}$ can, freely, be chosen to coincide with a symmetry mirror of the first tree $T_{1}$ (still perpendicular to $g$, of course). Then $m_{1}$ is fixed at distance half the translation amplitude of the glide along $g$ (not necessarily commensurate with any symmetry of the tree). Finally $m_{g}$ is reflection through the geodesic $g$ (Fig. 31).

Taking into account the symmetries of the regular trees, there is a point $c$ on $g$ which is the centre of a rotation by $\pi$ mapping $T_{1}$ onto $T_{2}$. Indeed the glide reflection of $T_{1}$ is $m_{g} m_{1} m_{2} T_{1}=m_{g} m_{1} T_{1}$, because $m_{2}$ is a symmetry of $T_{1}$. Now $R_{2}=m_{g} m_{1}$ is a two-fold rotation centred at the intersection $c$ of the lines $m_{1}$ and $g$.

Taking $m_{2}$ to be the perpendicular bisector of the edge common to $q$ and $T_{1}$, it follows that the joining quadrilateral $q$ is a parallelogram (its symmetry group is $\left\{1, R_{2}\right\}$ ). We can use this symmetry to split $q$, along geodesic $g$, into two congruent parts so that its area is twice the area of any of those half parts.

When the tree $T_{2}$ is shifted (along $g$ ) with respect to $T_{1}$, the quadrilateral is deformed (by a kind of hyperbolic shear), but its area does not change. This is proved in Figure 32: The deformed quadrilateral $\tilde{q}$ (or, rather, its fundamental half) can be transformed into $q$ by cut and paste of a triangle leaned against $g$. This proves A1.

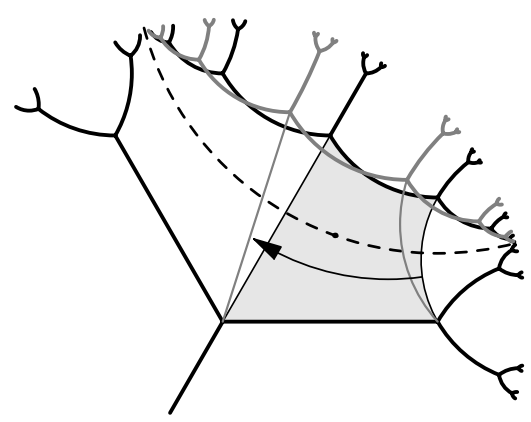

Fig. 32. Shifting one tree with respect to its neighbour induces a shear of the joining quadrilateral. Considering the half parts below the separating geodesic $g$, the difference between the two (deformed and undeformed quadrilaterals) appears to be a pair of triangles clearly translates of each other (by $t_{1}$ ).

\section{A.2 Independent of edge length}

To prove A2, we can take A1 into account (to shift the trees if necessary) and assume that the forest has maximal symmetry: the mirrors $m_{1}, m_{2}$ coincide. In this case, the half quadrilateral $q$ (which is still a quadrilateral) has angles $\pi / 2, \pi / 2, \pi / z, \pi / z$ at the vertices. So its area is

$$
\operatorname{area}(q)=2 \pi\left(1-\frac{2}{z}\right)
$$

(to check this, cut the half $q$ into two triangles by one of its diagonals; the angles are, respectively, $(\alpha, \beta, \pi / z)$ and $(\pi / z-\alpha, \pi / 2-\beta, \pi / 2)$; this yields areas equal to $\pi / 2-\alpha-\beta$ and $\pi(1 / 2-2 / z)+\alpha+\beta$ whose sum gives area $(q) / 2)$. This expression is indeed independent of $a$.

With equation (7), equation (A.1) provides an alternate derivation of equation (8).

\section{References}

1. A.F. Wells, Three-dimensional nets and polyhedra (John Wiley and Sons, 1977).

2. E. Koch, W. Fischer, Z. Kristallogr. 210, 407-414 (1995).

3. M. O'Keeffe, N.E. Breese, Acta Cryst. A 48, 663-669 (1992).

4. M. O'Keeffe, Acta Cryst. A 48, 670-673 (1992); 48, 879884 (1992); 51, 916-920 (1995).

5. M. O'Keeffe, B.G. Hyde, Crystal Structures. 1. Patterns and Symmetry Theory (Mineralogical Society of America, Washington, 1996).

6. O.D. Friedrichs, A.W. Dress, D.H. Huson, J. Klinowski, A.L. Mackay, Nature 400, 644-647 (1999).

7. M.M.J. Treacy, K.H. Randall, S. Rao, J.A. Perry, D.J. Chadi, Z. Kristallogr. 212, 768-791 (1997).

8. S.T. Hyde, S. Ramsden, Europhys. Lett. 50, 135-141 (2000).

9. S. R. Batten, R. Robson, Angew. Chem. Int. Ed. Engl. 37, 1460-1494 (1998).

10. L.E. Scriven, Nature 263, 123-125 (1976).

11. V. Luzzati, in Biological Membranes, edited by D. Chapman (Academic Press, 1968), pp. 71-123.

12. K. Larsson, Nature 304, 664 (1983). 
13. International Workshop on Geometry and Interfaces, edited by E. Dubois-Violette, B. Pansu, J. Phys. Colloq. France 51, C7 (1990).

14. J.C. Nitsche, Lectures on Minimal Surfaces (Cambridge, 1989).

15. U. Dierkes, S. Hildebrandt, A. Kuster, O. Wohlrab, Minimal Surfaces I 8 II, Grundl. der math. Wiss. Vol. 295 \& 296 (Springer, 1991).

16. S. Lidin, S.T. Hyde, B.W. Ninham, J. Phys. France 51, 801-813 (1990).

17. D. Hilbert, S. Cohn-Vossen, Geometry and the Imagination (Chelsea Publishers, New York, 1959).

18. H.S.M. Coxeter, W.O. Moser Generators and relations for discrete groups (Springer, 1957).

19. J.H. Conway, The orbifold notation for surface groups, in Lond. Math. Soc. Lecture Notes Series (Cambridge Univ. Press, 1992), pp. 438-447.

20. S.T. Hyde, S. Ramsden, in Chemical Topology, Series in Mathematical Chemistry Vol. 4, edited by D. Bonchev, D.H. Rouvray (Gordon \& Breach, 1999).

21. B. Söderberg, Phys. Rev. E 47, 4582 (1993).

22. H. Schwerdtfeger, Geometry of Complex Numbers (Dover, New York, 1979).

23. J.-F. Sadoc, J. Charvolin, Acta Cryst. A 45, 10-20 (1989).

24. M. Spivak A Comprehensive Introduction to Differential Geometry (Publish or Perish, Houston, 1993).

25. D.H. Huson, Two dimensional symmetry editing, DIMACS series in Discrete Maths and Theoretical Computer Sciences (in press, 1999).
26. A. Fogden, S.T. Hyde, Acta Cryst. A 48, 442-451; 575-591 (1990).

27. C. Oguey, J.-F. Sadoc, J. Phys. I France 3, 839-854 (1993).

28. H.S.M. Coxeter, Regular Polytopes (Dover, New York, 1973).

29. E. Koch, W. Fisher, Acta Cryst. A 46, 33-40 (1990).

30. S.T. Hyde, S. Ramsden, http://mesoscale.anu.edu.au

31. M. O'Keeffe, Z. Kristallogr. 210, 905-908 (1995).

32. R.W. Grosse-Kunsleve, G.O. Brunner, J.A. Sloane, Acta Cryst. A 52, 879-889 (1996).

33. J.H. Conway, J.A. Sloane, Proc. Roy. Soc. Lond. A 453, 2369-2389 (1997).

34. S.T. Hyde, Curr. Opinion Sol. State Mat. Sci. 1, 653-662 (1998).

35. S. Okamoto, H. Hasegawa, T. Hashimoto, T. Fujimoto, H. Zhang, T. Kazama, A. Takano, Y. Isono, Polymer 38, 5275-5281 (1997).

36. S. Sioula, N. Hadjichristidis, E.L. Thomas, Macromolecules 31, 5272-5277 (1998).

37. S. Sioula, N. Hadjichristidis, E.L.Thomas, Macromolecules 31, 8429-8432 (1998).

38. K.A. Hirsch, S.R. Wilson, J.S. Moore, Chem. Eur. J. 3, 765-771 (1997).

39. C. Kepert, M.J. Rosseinsky, Chem. Commun. 1998, 31-32 (1998).

40. B.F. Abrahams, S.R. Batten, H. Hamit, B.F. Hoskins, R. Robson, Chem. Commun. 1996, 1313-1314 (1996). 